\begin{tabular}{|c|c|}
\hline Title & Decreases in 15-lipoxygenase metabolites in OImsted syndrome model rats \\
\hline Author(s) & W akabay ashi, Masato; Y oshioka, Takeshi; Higashino, Kenichi; Numata, Y oshito; Igarashi, Y asuy uki; Kihara, A kio \\
\hline Citation & $\begin{array}{l}\text { Journal of dermatological science, 85(3), 186-196 } \\
\text { https://doi.org/0.1016/.jdermsci.2016.12.013 }\end{array}$ \\
\hline Issue Date & $2017-03$ \\
\hline Doc URL & http:/hdl.handle.net/2115/68655 \\
\hline Rights & $\begin{array}{l}\text { O201. Inis manuscript version is made avallable under the CC-BY-IVC-IVD } 4.0 \text { IIcense } \\
\text { http://reativecommons.org/icenses/by-nc-nd/4.0/ }\end{array}$ \\
\hline Rights(URL) & https://creativecommons.org/icenses/by-nc-nd/4.0/ \\
\hline Type & article (author version) \\
\hline File Information & WoS_79131_kihara.pdf \\
\hline
\end{tabular}

Instructions for use 


\section{Decreases in 15-lipoxygenase metabolites in Olmsted syndrome model}

rats

Masato Wakabayashi, ${ }^{\mathrm{a}, \mathrm{b}}$, Takeshi Yoshioka ${ }^{\mathrm{a}}$, Kenichi Higashino ${ }^{\mathrm{a}}$, Yoshito Numata ${ }^{\mathrm{a}}$, Yasuyuki Igarashic $^{\mathrm{c}}$, and Akio Kihara,d,*

${ }^{a}$ Shionogi Innovation Center for Drug Discovery, Discovery Research Laboratory for Innovative Frontier Medicines, Shionogi \& Co., Ltd., Sapporo 001-0021, Japan

${ }^{\mathrm{b}}$ Laboratory of Biochemistry, Graduate School of Life Science, Hokkaido University, Sapporo 060-0812, Japan

${ }^{\mathrm{c}}$ Laboratory of Biomembrane and Biofunctional Chemistry, Faculty of Advanced Life Science, Hokkaido University, Sapporo 001-0021, Japan

${ }^{\mathrm{d}}$ Laboratory of Biochemistry, Faculty of Pharmaceutical Sciences, Hokkaido University, Sapporo 060-0812, Japan

${ }^{*}$ Corresponding author.

Akio Kihara, $\mathrm{PhD}$

Laboratory of Biochemistry, Faculty of Pharmaceutical Sciences

Hokkaido University

Kita 12-jo, Nishi 6-chome, Kita-ku, Sapporo 060-0812, Japan

Tel: +81-11-706-3754

Fax: +81-11-706-4900

E-mail: kihara@pharm.hokudai.ac.jp 


\begin{abstract}
Background: Olmsted syndrome (OS) is a congenital dermatosis characterized by palmoplantar keratoderma and periorificial keratotic plaque. TRPV3 (transient receptor potential vanilloid subtype 3) encodes a thermosensitive $\mathrm{Ca}^{2+}$ channel and is the causative gene of OS. However, the molecular mechanism that causes the pathological development of OS is unclear.
\end{abstract}

Objective: We aimed to investigate the molecular mechanisms underlying OS pathology from the perspective of lipid metabolism.

Methods: Comprehensive lipidomics and microarray analyses were conducted on tissue samples from a non-lesional skin area of OS model rats (Ht rats) and from wild type (WT) rats as the control.

Results: Infiltration of leukocytes such as eosinophils and neutrophils and an increase in the fibrotic region were detected in the unaffected skin area of $H t$ rats compared with the WT rats. Among about 600 lipid species examined, the levels of 15-lipoxygenase (LOX) metabolites, the precursors of anti-inflammatory and pro-resolving lipid mediators, and dihydroceramides decreased by $\geq 16$-fold in Ht rats compared with WT rats. Consistent with the decreases in the 15-LOX metabolites, expression levels of the genes that encode the 15-LOXs, Alox15 and Alox15b, were largely reduced. Conversely, increased expression levels were detected of Il36b, Ccl20, Cxcl1, and Cxcl2, which encode cytokines/chemokines, and S100a8 and S100a9, which encode the $\mathrm{Ca}^{2+}$ binding proteins that are implicated in epidermal proliferation.

Conclusion: The pro-inflammatory state in the unaffected skin of $H t$ rats caused by decreases in 15-LOX metabolites and increases in cytokines/chemokines may contribute to the pathogenesis of OS. 
Keywords: Lipid; Lipidomics; 15-Lipoxygenase; Olmsted syndrome; TRPV3.

Abbreviations: OS, Olmsted syndrome; OMIM, Online Mendelian Inheritance in Man; Ht, WBN/Kob-Ht; WT, wild type; LOX, lipoxygenase; HE, hematoxylin-eosin; LC, liquid chromatography; MS, mass spectrometry; HETrE, hydroxyeicosatrienoic acid; HETE, hydroxyeicosatetraenoic acid; 15-oxETE, 15-oxoeicosatetraenoic acid; HDoHE, hydroxydocosahexaenoic acid; COX, cyclooxygenase; HEPE, hydroxyeicosapentaenoic acid; EOS, esterified $\omega$-hydroxyacyl-sphingosine. 


\section{Introduction}

Olmsted syndrome (OS; Online Mendelian Inheritance in Man (OMIM) \#614594) is a severe keratinization disorder characterized by the combination of palmoplantar and periorificial keratoderma and is often associated with hypotrichosis and intense itching [1]. OS is a very rare congenital disorder, and only 73 cases have been reported so far [1]. Most of the reported OS cases were sporadic; however, some familial cases with different hereditary patterns were also found. The hereditary patterns were either autosomal or X-linked and either dominant or recessive [1]. TRPV3 (transient receptor potential vanilloid subtype 3) on chromosome 17 and MBTPS2 (membrane-bound transcription factor protease, site 2) on chromosome $\mathrm{X}$ have been identified as the causative genes of OS [2,3]. TRPV3 is a thermosensitive $\mathrm{Ca}^{2+}$ channel [4-6]. Most of the TRPV3 mutations (p.Gly573Ser, p.Gly573Cys, p.Gly573Ala, p.Gln580Pro, p.Leu673Phe, p.Trp692Gly, and p.Trp692Cys) found in OS patients were dominant [1], and expression of the mutant proteins caused increases in intracellular $\mathrm{Ca}^{2+}$ concentrations [2,7]. Recessive mutations in TRPV3 have also been identified for OS $[1,8]$. The reason these diverse inheritance patterns exist for OS is currently unclear. The MBTPS2 mutations found in OS patients were recessive [3,9]. MBTPS2 is a component of the regulated intramembrane proteolysis machinery that regulates cholesterol homeostasis and the unfolded protein response by cleaving membrane-spanning regulatory proteins [10].

TRPV3 belongs to the vanilloid family of transient receptor cation channels [11]. TRPV3 was shown to be highly expressed in skin, primarily in hair follicles and in keratinocytes that are present in the basal layer of the epidermis, and its expression has also been detected in brain, spinal cord, and dorsal root ganglion [5,6]. TRPV3 is 
activated by temperature, with a threshold of $31-39^{\circ} \mathrm{C}$ [4-6], as well as by ligands such as 2-aminoethoxydiphenyl borate and farnesyl pyrophosphate, which is an intermediate metabolite of the mevalonate pathway [12,13]. Furthermore, arachidonic acid and other unsaturated fatty acids were reported to potentiate TRPV3 activity [14], while the pro-resolving lipid mediator $17(R)$-resolvin D1 was found to inhibit TRPV3 activity [15]. Thus, there appears to be a relationship between TRPV3 activity and lipids.

WBN/Kob-Ht rats (hereafter called $H t$ rats) and DS-Nh mice were selected as spontaneous hairless rodent strains, and both strains had dominant Trpv3 mutations (p.Gly573Cys in $H t$ rats and p.Gly573Ser in DS-Nh mice), the same as two of the mutations found in OS patients [16]. These rodents developed dermatitis accompanied with hyperkeratosis and acanthosis under conventional conditions [17,18]. The p.Gly573Ser transgenic mice also showed dermatitis with severe itching and defects in hair growth [19]. However, OS-like phenotypes were not observed in Trpv3 knockout mice; rather, they exhibited thin stratum corneum, wavy whiskers, and misaligned hair follicles [20,21]. These results suggest that OS is caused by gain-of-function of TRPV3.

The skin permeability barrier is important for the prevention of loss of water and electrolytes from inside the body and for protection against the invasion of external pathogens, allergens, and harmful compounds. Multi-layered lipids, called lipid lamellae, are present in the outermost cell layer of the epidermis (stratum corneum), where they have a pivotal function in skin permeability barrier formation [22]. The major constituents of lipid lamellae are ceramides (about 50\%), cholesterol (about 25\%), and free fatty acids (about 15\%) [23,24]. Ceramides form the backbones of sphingolipids, and a variety of ceramide species are present in the epidermis [22,24] (Fig. S1). Acylceramide is the epidermis-specific ceramide and is especially important 
for skin permeability barrier formation [22]. Normal ceramide consists of a long-chain base and an amide-linked fatty acid, whereas acylceramide contains an extra linoleic acid attached to the $\omega$-carbon of the amide-linked fatty acid [22,24] (Fig. S1). Mutations in genes involved in acylceramide synthesis cause autosomal recessive congenital ichthyosis, which is characterized by severe skin permeability barrier defects, scaly skin, and hyperkeratosis [24,25].

Considering the importance of lipids in skin permeability barrier formation and the regulatory roles of lipids on TRPV3 activity, it is likely that changes in lipid metabolism contribute to OS pathology. However, lipidomics analyses of skin tissues of OS patients or OS model animals have not been performed so far, although transcriptome and proteome analyses have already been reported [7]. Here, we performed comprehensive comparative lipidomics analyses of skin samples from non-lesional areas of wild type (WT) and Ht rats to reveal the lipid metabolism underlying the OS pathology. 


\section{Methods}

\subsection{Animals}

WBN/Kob (WT) and WBN/Kob-Ht (Ht; Trpv3 p.Gly573Cys) rats were purchased from the Ishikawa Laboratory Animal Company (Saitama, Japan) and housed under conventional conditions under a 12-h light/dark cycle with standard food and water ad libitum. All animal experiments were conducted according to the guidelines for animal experimentation at Shionogi \& Co., Ltd. (Osaka, Japan). Skin and brain tissue samples were taken from WT and $H t$ rats at 25 weeks of age, frozen immediately, and stored at $-80^{\circ} \mathrm{C}$ until used for analyses.

\subsection{Histological analyses}

Skin samples were fixed with 10\% neutral pH-buffered formaldehyde (Wako Pure Chemical Industries, Osaka, Japan) for 2 days and embedded in paraffin. Paraffin-embedded skins were cut into 3-4- $\mu$ m sections, deparaffinized, and subjected to hematoxylin-eosin (HE), toluidine blue, Masson's trichrome, and Luna stainings $[26,27]$.

\subsection{Hydroxyproline measurement}

Hydroxyproline levels were measured using a Hydroxyproline Assay Kit (Quickzyme, Leiden, Netherlands), according to the manufacturer's instructions.

\subsection{Measurement of myeloperoxidase activity}

Myeloperoxidase activity was measured using an OxiSelect Myeloperoxidase

Chlorination Activity Assay Kit (Cell Biolabs, San Diego, CA), according to the 
manufacturer's instructions.

\subsection{Transmission electron microscopy}

Samples taken from the non-lesional dorsal skin of WT and Ht rats at 3 weeks of age were fixed with $4 \%$ paraformaldehyde and $2 \%$ glutaraldehyde in $0.1 \mathrm{M}$ cacodylate buffer (pH 7.4) at $4^{\circ} \mathrm{C}$ and then with $0.1 \%$ tannic acid in $0.1 \mathrm{M}$ cacodylate buffer (pH 7.4) at $4^{\circ} \mathrm{C}$. Fixed samples were washed four times with $0.1 \mathrm{M}$ cacodylate buffer (pH 7.4), followed by post-fixation with $2 \%$ osmium tetroxide in $0.1 \mathrm{M}$ cacodylate buffer $(\mathrm{pH} 7.4)$ at $4^{\circ} \mathrm{C}$. The samples were then dehydrated in graded ethanol solutions $(50 \%$, 70\%, 90\%, and 100\%), followed by infiltration with propylene oxide for $1 \mathrm{~h}$ twice, and finally with a propylene oxide/Quetol-812 resin (Nisshin EM, Tokyo, Japan) mixture (7:3, v/v) for $1 \mathrm{~h}$. After the propylene oxide was volatilized overnight, the samples were embedded in fresh $100 \%$ resin and incubated at $60^{\circ} \mathrm{C}$ for $48 \mathrm{~h}$. Ultra-thin sections (80 $\mathrm{nm}$ ) were prepared using the ultramicrotome Ultracut UCT (Leica Microsystems, Wetzlar, Germany). The sections were mounted on copper grids, stained with $2 \%$ uranyl acetate at room temperature for 15 min, washed with distilled water, and secondary-stained with Lead stain solution (Sigma-Aldrich, St. Louis, MO) at room temperature for 3 min. Microscopy was performed using a JEM-1400Plus transmission electron microscope (JEOL, Tokyo, Japan) at an acceleration voltage of $80 \mathrm{kV}$. Digital images were taken using a VELETA charge-coupled device camera (Olympus, Tokyo, Japan).

2.6. Lipid analyses by liquid chromatography (LC) coupled with mass spectrometry $(M S)$ 
Details of the lipid extraction and lipid analyses by LC/MS are described in the Supplementary Information and Tables S1-S4.

\subsection{Microarray analysis}

Total RNAs were extracted from skin samples using TRIzol reagent (Thermo Fisher Scientific) and purified using an RNeasy Mini kit (Qiagen, Hilden, Germany). The purified RNAs were converted to cRNAs and labeled with Cy3 using a Low Input Quick Amp Labeling Kit, One-Color (Agilent Technologies). The cRNAs were fragmented using a Gene Expression Hybridization Kit (Agilent Technologies) and hybridized to a SurePrint G3 Rat GE $8 \times 60 \mathrm{~K}$ v2 microarray (Agilent Technologies) at $65^{\circ} \mathrm{C}$ for $17 \mathrm{~h}$. The microarray slide was washed and dried, and then scanned using a SureScan microarray scanner G2600D (Agilent Technologies). The raw microarray image data were analyzed with Feature Extraction software 11.5.1.1 (Agilent Technologies). Gene functions were predicted using the web-based tool GeneMANIA (http://www.genemania.org/) [28]. The microarray data have been deposited in the Gene Expression Omnibus database at http://www.ncbi.nlm.nih.gov/geo/ under accession number GSE84294.

\subsection{Real-time RT-PCR}

Total RNAs were extracted from skin samples using TRIzol reagent (Thermo Fisher Scientific) and purified using an RNeasy Mini kit (Qiagen). The purified RNAs were converted to cDNAs using a PrimeScript RT Reagent Kit (Takara Bio, Shiga, Japan). Real-time RT-PCR was performed using SYBR premix Ex Taq II (Tli RNaseH Plus) (Takara Bio) and gene-specific primers (Table S5) on a 7500 Real-time PCR 
system (Life Technologies).

\subsection{Statistical analyses}

Comparisons between two groups were performed using the Wilcoxon rank sum test or Student's t-test. SAS v9.2 software (SAS Institute, Cary, NC) was used for all the statistical analyses. 


\section{Results}

\subsection{Leukocyte infiltration and fibrotic region increase in unaffected skin area of Ht rats}

OS model rats (Ht rats) have the p.Gly573Cys mutation in Trpv3 and exhibit atopic dermatitis-like skin symptoms, increases in infection of Staphylococcus aureus, and infiltration of mast cells, eosinophils, and T lymphocytes in the affected area $[16,18]$. However, how such changes are induced remains unclear. To understand the molecular events that trigger the OS pathology, we performed histological and biochemical analyses using non-lesional skin areas of $H t$ rats. HE staining revealed hyperkeratosis, parakeratosis, acanthosis, and infiltration of granulated leukocytes and monocytes in the affected area of the dorsal skin of the $H t$ rats, as described previously [16,18] (Fig. S2). No such prominent histopathological abnormalities were detected in the unaffected dorsal skin tissue of the $H t$ rats, although reduced hair shaft and irregularity of the hair follicle position were observed (Fig. 1A). Toluidine blue and Luna stainings showed that the numbers of mast cells and eosinophils were increased even in the unaffected skin area of the Ht rats compared with WT rats (Fig. 1A). Furthermore, the dermis of the $H t$ rats was more highly stained with Masson's trichrome than the dermis of the WT, indicating that collagen was increased in the $H t$ rats. This result was confirmed by the higher hydroxyproline levels measured in the unaffected skin areas of $\mathrm{Ht}$ rats compared with the WT (Fig. 1B). Myeloperoxidase activity, which is the hallmark of neutrophil infiltration, was also increased in the unaffected skin area of the $H t$ rats compared with the WT (Fig. 1C). We also examined the skin samples using electron microscopy. The number of stratum corneum layers was slightly higher in $H t$ rats compared with WT (Fig. 1D). Therefore, although overall histological abnormalities were not observed in the unaffected skin area of the Ht rats, infiltration of leukocytes and increases in fibrotic 
regions and stratum corneum layers were detected.

\subsection{5-Lipoxygenase (LOX) metabolites decrease in the unaffected skin area of Ht rats}

Lipidomics analyses have not been performed on OS patients or model animals until now, even though a possible relationship between lipids and TRPV3 activity has been considered and the importance of lipids in skin barrier formation and inflammation is well known. We performed comprehensive lipidomics analyses on the unaffected skin area of $\mathrm{Ht}$ rats and detected approximately 600 lipid species ranging from fatty acids, fatty acid metabolites, glycerolipids, sphingolipids, and cholesterol using a combination of non-targeted and targeted LC/MS analyses (Tables S6 and S7). Among the lipid species, we found that 15-LOX metabolites (15-hydroxyeicosatrienoic acid (HETrE), 15-hydroxyeicosatetraenoic acid (HETE), 15-oxoeicosatetraenoic acid (15-oxETE), and 17-hydroxydocosahexaenoic acid (HDoHE)) and dihydroceramides were largely decreased in $\mathrm{Ht}$ rats compared with the WT (Fig. 2A).

The precursors of the 15-LOX metabolites such as dihomo- $\gamma$-linolenic acid, arachidonic acid, and docosahexaenoic acid were also lower in $\mathrm{Ht}$ rats than in WT rats (Fig. 2B). In the skin of WT rats, 15-LOX metabolites were much more abundant than other oxidized fatty acids such as cyclooxygenase (COX) metabolites, 5-LOX metabolites, and 12-LOX metabolites (Fig. 2C-F). Among these oxidized fatty acid metabolites (Fig. S3), decreases in 15-LOX metabolites (15-HETrE, 15-HETE, 15-oxETE, 15-hydroxyeicosapentaenoic acid (HEPE), and 17-HDoHE) were most prominent in the $\mathrm{Ht}$ rats, although some decreases in the COX metabolite prostaglandin $\mathrm{D}_{2}$, 5-LOX metabolites (5-HETE, 5-HEPE, and 7-HDoHE), and 12-LOX metabolites (12-HETE, 12-HEPE, and 14-HDoHE) were also observed (Fig. 2C-F). 
Ceramides (broad definition) include several ceramide species such as the dihydroceramides that have dihydrosphingosine as a long-chain base, ceramides (narrow definition) that have sphingosine, phytoceramides that have phytosphingosine, and acylceramides [22] (Fig. S1). Although dihydrosphingosine is saturated, sphingosine has a trans-double bond. Phytosphingosine exists in specific tissues, including the epidermis, and contains an additional hydroxyl group at the C4 position. In $H t$ rats, most of the dihydroceramides, especially d18:0/18:0 and d18:0/20:0 dihydroceramides, were largely decreased compared with those in WT rats (Fig. 2G). Because d18:0/18:0 and d18:0/20:0 dihydroceramides were abundant in hair [29], the observed decreases may reflect the hypotrichosis phenotype of the Ht rats. In comparison, the changes in the levels of phytoceramides, ceramides (narrow definition), and acylceramides (esterified $\omega$-hydroxyacyl-sphingosines; EOSs) were rather modest (Fig. 2G and Table S7). In summary, the lipidomics analyses revealed striking decreases in the levels of 15-LOX metabolites and dihydroceramides in the unaffected skin area of the $H t$ rats compared with the WT. As the decreases in dihydroceramides were anticipated because of the hypotrichosis phenotype, we focused on the 15-LOX metabolites in the further analyses.

TRPV3 is expressed in the stratum basale of epidermis and hair follicles [5]. To investigate whether these tissues produce 15-LOX metabolites, dorsal skin of WT rats was separated into two parts: upper (hair and epidermis) and lower (dermis, subcutaneous tissues, and muscle). Lipids extracted from each part were then subjected to LC/MS analyses. We found that the four 15-LOX metabolites examined were highly concentrated in the upper portion of the skin (Fig. 3A). The levels of three 12-LOX metabolites were also higher in the upper portion, but only slightly (Fig. 3B). Moreover, 
three 5-LOX metabolites and four COX metabolites were distributed in both skin portions (Fig. 3C and D). These results suggest that epidermis and hair are responsible for the observed decreases in the levels of 15-LOX metabolites in the unaffected skin area of the Ht rats.

\subsection{5-LOX metabolites are not decreased in Ht rat brain}

Besides skin, TRPV3 is also expressed in the brain [6]. Therefore, we examined whether the OS p.Gly573Cys mutation in Trpv3 affected the levels of 15-LOX metabolites and dihydroceramides in the brain. We found that the levels of 15-LOX metabolites and dihydroceramides were similar in the brain tissue of $H t$ and WT rats (Fig. 4). Therefore, the effect of the enhanced TRPV3 activity on lipid metabolism in the brain is likely to be small. This result is consistent with the symptoms of OS, which are not associated with aberrations of the central nervous system [30]. Thus, the decreases in 15-LOX metabolites and dihydroceramides as a result of the OS mutation may be confined to skin.

\subsection{Expression levels of 15-LOX genes are decreased in Ht rat skin}

To gain insights into the causes of the differences in lipid profiles between WT and $H t$ rat skin, we performed microarray gene expression analyses using unaffected skin. The gene expression results were classified as follows: i) genes with decreased expression in $H t$ rats (Table 1), ii) genes with increased expression in $H t$ rats (Table 2), and iii) highly expressed genes in $H t$ rats among genes exhibiting increased expression (fold increase $\left(\log _{2} H t / \mathrm{WT}\right)>1.5$; Table 3). The top 30 genes in each category are presented in Tables 1-3. The expression levels of a number of genes related to keratins, 
which form the cell cytoskeleton and hair shaft, were decreased in Ht rats compared with the WT (Table 1). The expression levels of the $\mathrm{Ca}^{2+}$ binding protein-encoding gene S100a9 and the chemokine-encoding genes Ccl20, Cxcl1, and Cxcl2 were increased in Ht rats compared with the WT (Table 2). S100a9 and S100a8 form homo- or heterodimers and are involved in regulating the responses to epidermal injury, inflammation, and disease [31]. The expression of S100a8 was also elevated in $\mathrm{Ht}$ rats compared with the WT (Table 3). The increased abundance of chemokines may be related to the infiltration of leukocytes, as shown in Fig. 1. Furthermore, the expression levels of antibacterial peptide Defb1, cytokine Il36b encoding IL-36ß, fatty acid elongase Elovl3, 2-hydroxyacyl-CoA lyase Hacl1, acyl-CoA wax alcohol acyltransferase 1 Awat1, and isopentenyl-diphosphate $\Delta$-isomerase 1 Idi1 were also elevated (Table 3). The IL-36 cytokine family induces proinflammatory mediators TNF- $\alpha$, IL-6, and IL-8 and seems to be involved in psoriasis [32]. Elovl3 is responsible for the production of C20 acyl-CoA [33,34]. Hacl1 is involved in the metabolism of 2-hydroxyfatty acids, which are abundant in epidermal ceramides [22,35]. Awat1 is responsible for the production of wax ester, which is secreted from sebaceous glands [36]. Idi1 is involved in the synthesis of isoprenoids [37].

We used GeneMANIA (http://www.genemania.org/) to predict gene function and analyze the gene lists obtained from the microarray analyses [28]. The predicted functions were mainly cell cytoskeleton formation for the genes with decreased expression in $\mathrm{Ht}$ rats (Fig. S4), cell migration of leukocytes for genes with increased expression in $\mathrm{Ht}$ rats (Fig. S5), and lipid metabolism for highly expressed genes among genes exhibiting increased expression in Ht rats (Fig. S6).

To examine the changes in gene expression related to LOX and COX metabolites 
and dihydroceramides, we performed real-time RT-PCR using unaffected skin of WT and $H t$ rats. In rats, 12/15-LOX-1 encoded by Alox15 was reported to have both 12-LOX and 15-LOX activities, although the 15-LOX activity was low [38]. However, 12/15-LOX-2 encoded by Alox15b showed only 15-LOX activity [38]. The expression levels of both Alox15 and Alox15b were largely decreased in Ht rats compared with the WT (Fig. 5), suggesting that the decreases in 15-LOX metabolites (Fig. 2B) were caused by decreases in the expression levels of 15-LOX genes. The expression levels of 5-LOX (Alox5), 12-LOXs (Alox12b and Alox12e), and COX-2 (Ptgs2) were almost the same in $H t$ and WT rats. The sphingolipid $\Delta 4$-desaturase DEGS1 catalyzes the introduction of a trans-double bound between positions 4 and 5 of dihydrosphingosine in dihydroceramide, and thus converts dihydroceramides to ceramides (narrow definition) [39]. Although a 1.6-fold increase in the Degs1 expression level was observed in $\mathrm{Ht}$ rats, this small increase does not explain the large decreases in dihydroceramides (Fig. 2G). The expression level of Cers3, which encodes the ceramide synthase that is involved in the synthesis of C18-C36 (dihydro)ceramides [22] was slightly increased, whereas the expression level of Cers4, which encodes the ceramide synthase that is involved in the synthesis of C18-22 (dihydro)ceramides [22] was not significantly increased in Ht rats compared with the WT (Fig. 5). These changes in expression levels are inconsistent with the decreases in C16-C22 dihydroceramides (Fig. 2G). The expression levels of Elovl1, which encodes the fatty acid elongase that is involved in the production of C20- to C26-CoAs [27,33], and of Sgms1 and Sgms2, which encode sphingomyelin synthases, were similar in Ht and WT rats (Fig. 5). The expression level of Elovl4, which encodes the fatty acid elongase that is involved in the production of $\geq \mathrm{C} 24$-CoAs [33], was increased in $\mathrm{Ht}$ rats (Fig. 5). Consistent with the 
microarray data, the expression levels of Elovl3, Cxcl1, Cxcl2, Ccl2, and Il36b were found to be increased in Ht rats compared with the WT. The data obtained from the gene expression analyses, which showed decreases in the expression levels of 15-LOX genes and increases in the expression levels of chemokine/cytokine genes, provide insights into the causes of histological and lipid metabolic changes, such as leukocyte infiltration and decreases in 15-LOX metabolites in Ht rats.

We next examined expression levels of Alox15 and Alox15b in brain. The expression levels of Alox15 were much higher than those of Alox15b (Fig. S7). Therefore, Alox15 seems to be mainly responsible for production of 15-LOX metabolites in the brain. Although the expression levels of Alox15b in brain were decreased in $H t$ rats compared with WT rats, those of Alox15 were similar. These data are consistent with the results that the levels of 15-LOX metabolites such as 15-HETrE and 15-HETE were similar in brains of WT and $\mathrm{Ht}$ rats (Fig. 4A). 


\section{Discussion}

To understand the molecular mechanism underlying OS syndrome, we performed comprehensive lipidomics analyses and detected approximately 600 lipid species using unaffected skin from OS model $(\mathrm{Ht})$ and WT rats. We found large decreases in the levels of 15-LOX metabolites and dihydroceramides in the unaffected skin area of $\mathrm{Ht}$ rats (Fig. 2). Although dihydroceramides exist only at low levels in most mammalian tissues, they are exceptionally abundant in hair [29]. Among dihydroceramide species, we observed the largest decrease in d18:0/20:0 dihydroceramide in Ht rats (Fig. 2G). The ceramide synthase CerS4 is mainly involved in the production of d18:0/20:0 dihydroceramide [22], and CerS4 knockout mice exhibit the hypotrichosis phenotype [40], suggesting the importance of d18:0/20:0 dihydroceramide in hair production and/or maintenance. Real time RT-PCR analyses revealed that the expression of Degs1, which is involved in conversion of dihydroceramide to ceramide, increased slightly in $H t$ rats, whereas expression of Cers4 was nearly unchanged (Fig. 5). The slight increase in Degs1 expression may not be enough to explain the large decreases in dihydroceramide levels that we found in $H t$ rats. Rather, it is likely that the large decreases in dihydroceramides in the unaffected skin area of $\mathrm{Ht}$ rats were instead due to hypotrichosis (Fig. 1A). It has been reported that activation of TRPV3 in outer root sheath keratinocytes of hair follicles induces elevated intracellular $\mathrm{Ca}^{2+}$ concentrations, which in turn inhibited proliferation and induced apoptosis of the keratinocytes [41].

15-LOXs catalyze the production of fatty acid hydroperoxides, which are rapidly reduced to hydroxides, including 15-HETrE derived from dihomo- $\gamma$-linolenic acid, 15-HETE from arachidonic acid, 15-HEPE from eicosapentaenoic acid, and 17-HDoHE from docosahexaenoic acid (Fig. S3). We found that all these hydroxides were almost 
undetectable in the unaffected skin area of $\mathrm{Ht}$ rats (Fig. 2F). The decreases in the 15-LOX metabolites were consistent with the decreased expression of Alox15 and Alox15b, the genes involved in their synthesis (Fig. 5). Human ALOX15B is expressed in prostate, lung, skin, and cornea [42]. In skin, $A L O X 15 B$ expression was detected in sebaceous, apocrine, and eccrine glands and in the basal layer of the epidermis [43]. In prostate, $A L O X 15 B$ was found to be involved in differentiation, senescence, and growth of prostate epithelial cells [44].

The 15-LOX hydroxide metabolites are converted to secondary lipid mediators such as lipoxin $\mathrm{A}_{4}$ from 15-HETE and protectin D1/resolvin D1 from 17-HDoHE [45] (Fig. S3). Importantly, all of these secondary lipid mediators have anti-inflammatory and pro-resolving properties [46-48]. Lipoxin $\mathrm{A}_{4}$ inhibits chemotaxis of neutrophils and eosinophils and stimulates monocyte chemotaxis and macrophage phagocytosis [49-52]. Protectin D1 and resolvin D1 also inhibit neutrophil infiltration and stimulate non-inflammatory phagocytosis of apoptotic neutrophils by macrophages [46]. Therefore, it is highly likely that the infiltration of eosinophils and neutrophils that we observed in the unaffected skin area of $H t$ rats (Fig. 1) was at least partly caused by decreases in these 15-LOX metabolite-derived lipid mediators.

We observed increased collagen levels, which is indicative of fibrosis, in the unaffected skin areas of $\mathrm{Ht}$ rats (Fig. $1 \mathrm{~A}$ and B). Fibrosis is induced by chronic inflammation caused by various stimuli, including persistent infections, allergic responses, radiation exposure, and tissue injury [53]. The affected skin of $H t$ rats exhibited atopic dermatitis-like skin symptoms, increases in S. aureus infections, and infiltration of mast cells, eosinophils, and T lymphocytes [16,18]. Here, we observed infiltration of leukocytes such as mast cells, eosinophils, and neutrophils, even in the 
unaffected skin of $H t$ rats (Fig. 1A and 1C). Thus, we speculate that inflammation in the unaffected skin of $H t$ rats caused mild fibrosis.

Microarray analyses also indicated the pro-inflammatory state of the unaffected skin area of Ht rats. For example, expression levels of the chemokine-encoding genes Ccl20, Cxcl1, and Cxcl2 were increased (Table 2). Cxcl1 and Cxcl2 stimulate chemotaxis of neutrophils and endothelial cells, and Ccl20 promotes chemotaxis of T and B cells [54]. Expression of Il36b, which encodes the cytokine IL-36 $\beta$, was also elevated in Ht rat skin (Table 2). IL-36 cytokines include IL-36 $\alpha$, IL-36 $\beta$, and IL-36 $\gamma$, which activate the IL-36 receptor, and IL36Ra, which acts as an antagonist [55]. IL-36 cytokines are expressed predominantly in keratinocytes and are important for regulation of skin inflammation [55]. Transgenic mice expressing IL-36 $\alpha$ in basal keratinocytes were reported to exhibit a psoriasis-like skin phenotype accompanying acanthosis and hyperkeratosis [56].

Microarray analyses revealed that the expression levels of the $\mathrm{Ca}^{2+}$ binding protein-encoding genes S100a8 and S100a9 were increased in Ht rat skin (Tables 2 and 3). S100a8 and S100a9 are members of the S100 protein family and form homo- and heterodimers [31]. S100 proteins are mediators of $\mathrm{Ca}^{2+}$-associated signal transduction, and they have been implicated in the pathogenesis of several epidermal diseases [31]. S100a8 and S100a9 are present at low levels in normal skin; however, their abundances were found to increase in skin under pathological and stressed conditions such as psoriasis, epidermal injury, and ultraviolet irradiation [57-59], suggesting S100a8 and S100a9 play a role in epidermal proliferation [31]. TRPV3 is a $\mathrm{Ca}^{2+}$ channel; therefore, the gain-of-function mutation causing OS syndrome elevates intracellular $\mathrm{Ca}^{2+}$ concentrations $[2,7]$. Considering that S100a8 and S100a9 are $\mathrm{Ca}^{2+}$ binding proteins, it 
is possible that elevated $\mathrm{Ca}^{2+}$ concentrations directly activate S100a8 and S100a9, leading to the epidermal proliferation that has been observed in the affected skin area of OS patients and model animals $[1,18]$

OS patients suffer from keratotic lesions on the palms, soles, and periorificial (mouth, nose, eyes, ears, genital, and anal) regions [1]. Conversely, $\mathrm{Ht}$ rats and DS-Nh mice develop skin symptoms on the neck, shoulder, and lateral/perioral areas of the face $[17,18]$. Although there are differences between OS patients and rodent models in where the symptoms appear, the types of skin symptoms, such as bacterial infection, infiltration of leukocytes, and itching, are similar. Hence, rodent models are useful for investigation of OS pathogenesis. The differences in symptomatic regions may be caused by different environmental conditions; for example, in rodent models, animals are maintained under fixed temperature, humidity, and diet, whereas these factors would be variable for OS patients. OS patients are therefore likely to be influenced by various exogenous stimuli that would not apply to rodents living in a restricted environment, including physical pressure, infection, allergens, and variable diet, temperature, and humidity.

In the present study, we showed that 15-LOX metabolites, which are the precursors of anti-inflammatory and pro-resolving lipid mediators, were decreased in the unaffected skin area of the OS model rats by comprehensive lipidomics analyses. Furthermore, subsequent microarray analyses demonstrated that some pro-inflammatory chemokines/cytokines were elevated. From these results, we speculate that the pro-inflammatory state plays a role in triggering the pathogenesis of OS syndrome (Fig. 6). Our findings may provide insights into the future development of OS therapy, such as using 15-LOX metabolites or their derivatives. 


\section{Acknowledgement}

We thank Ichiro Hikita for useful discussions. This research was supported by the Creation of Innovation Centers for Advanced Interdisciplinary Research Areas Program, Ministry of Education, Culture, Sports, Science and Technology, Japan, and by a grant from Shionogi \& Co., Ltd, Japan.

\section{Conflicts of interest}

The authors have no conflicts of interest to declare. 


\section{References}

[1] S. Duchatelet, A. Hovnanian, Olmsted syndrome: clinical, molecular and therapeutic aspects, Orphanet J. Rare Dis. 10 (2015) 33.

[2] Z. Lin, Q. Chen, M. Lee, X. Cao, J. Zhang, D. Ma, L. Chen, X. Hu, H. Wang, X. Wang, P. Zhang, X. Liu, L. Guan, Y. Tang, H. Yang, P. Tu, D. Bu, X. Zhu, K. Wang, R. Li, Y. Yang, Exome sequencing reveals mutations in TRPV3 as a cause of Olmsted syndrome, Am. J. Hum. Genet. 90 (2012) 558-564.

[3] A. Haghighi, C.A. Scott, D.S. Poon, R. Yaghoobi, N. Saleh-Gohari, V. Plagnol, D.P. Kelsell, A missense mutation in the MBTPS2 gene underlies the X-linked form of Olmsted syndrome, J. Invest. Dermatol. 133 (2013) 571-573.

[4] G.D. Smith, M.J. Gunthorpe, R.E. Kelsell, P.D. Hayes, P. Reilly, P. Facer, J.E. Wright, J.C. Jerman, J.P. Walhin, L. Ooi, J. Egerton, K.J. Charles, D. Smart, A.D. Randall, P. Anand, J.B. Davis, TRPV3 is a temperature-sensitive vanilloid receptor-like protein, Nature 418 (2002) 186-190.

[5] A.M. Peier, A.J. Reeve, D.A. Andersson, A. Moqrich, T.J. Earley, A.C. Hergarden, G.M. Story, S. Colley, J.B. Hogenesch, P. McIntyre, S. Bevan, A. Patapoutian, A heat-sensitive TRP channel expressed in keratinocytes, Science 296 (2002) 2046-2049.

[6] H. Xu, I.S. Ramsey, S.A. Kotecha, M.M. Moran, J.A. Chong, D. Lawson, P. Ge, J. Lilly, I. Silos-Santiago, Y. Xie, P.S. DiStefano, R. Curtis, D.E. Clapham, TRPV3 is a calcium-permeable temperature-sensitive cation channel, Nature 418 (2002) 181-186.

[7] Y. He, K. Zeng, X. Zhang, Q. Chen, J. Wu, H. Li, Y. Zhou, G. Glusman, J. Roach, A. Etheridge, S. Qing, Q. Tian, I. Lee, X. Tian, X. Wang, Z. Wu, L. Hood, Y. Ding, K. 
Wang, A gain-of-function mutation in TRPV3 causes focal palmoplantar keratoderma in a Chinese family, J. Invest. Dermatol. 135 (2015) 907-909.

[8] O. Eytan, D. Fuchs-Telem, B. Mevorach, M. Indelman, R. Bergman, O. Sarig, I. Goldberg, N. Adir, E. Sprecher, Olmsted syndrome caused by a homozygous recessive mutation in TRPV3, J. Invest. Dermatol. 134 (2014) 1752-1754.

[9] H.J. Wang, Z.L. Tang, Z.M. Lin, L.L. Dai, Q. Chen, Y. Yang, Recurrent splice-site mutation in MBTPS2 underlying IFAP syndrome with Olmsted syndrome-like features in a Chinese patient, Clin. Exp. Dermatol. 39 (2014) 158-161.

[10] G. Chen, X. Zhang, New insights into S2P signaling cascades: regulation, variation, and conservation, Protein Sci. 19 (2010) 2015-2030.

[11] B. Nilius, T. Biro, G. Owsianik, TRPV3: time to decipher a poorly understood family member!, J. Physiol. 592 (2014) 295-304.

[12] M.K. Chung, H. Lee, A. Mizuno, M. Suzuki, M.J. Caterina, 2-Aminoethoxydiphenyl borate activates and sensitizes the heat-gated ion channel TRPV3, J. Neurosci. 24 (2004) 5177-5182.

[13] S. Bang, S. Yoo, T.J. Yang, H. Cho, S.W. Hwang, Farnesyl pyrophosphate is a novel pain-producing molecule via specific activation of TRPV3, J. Biol. Chem. 285 (2010) 19362-19371.

[14] H.Z. Hu, R. Xiao, C. Wang, N. Gao, C.K. Colton, J.D. Wood, M.X. Zhu, Potentiation of TRPV3 channel function by unsaturated fatty acids, J. Cell. Physiol. 208 (2006) 201-212.

[15] S. Bang, S. Yoo, T.J. Yang, H. Cho, S.W. Hwang, 17(R)-resolvin D1 specifically inhibits transient receptor potential ion channel vanilloid 3 leading to peripheral antinociception, Br. J. Pharmacol. 165 (2012) 683-692. 
[16] M. Asakawa, T. Yoshioka, T. Matsutani, I. Hikita, M. Suzuki, I. Oshima, K. Tsukahara, A. Arimura, T. Horikawa, T. Hirasawa, T. Sakata, Association of a mutation in TRPV3 with defective hair growth in rodents, J. Invest. Dermatol. 126 (2006) 2664-2672.

[17] I. Hikita, T. Yoshioka, T. Mizoguchi, K. Tsukahara, K. Tsuru, H. Nagai, T. Hirasawa, Y. Tsuruta, R. Suzuki, M. Ichihashi, T. Horikawa, Characterization of dermatitis arising spontaneously in DS-Nh mice maintained under conventional conditions: another possible model for atopic dermatitis, J. Dermatol. Sci. 30 (2002) 142-153.

[18] M. Asakawa, T. Yoshioka, I. Hikita, T. Matsutani, T. Hirasawa, A. Arimura, T. Sakata, T. Horikawa, WBN/Kob-Ht rats spontaneously develop dermatitis under conventional conditions: another possible model for atopic dermatitis, Exp. Anim. 54 (2005) 461-465.

[19] T. Yoshioka, K. Imura, M. Asakawa, M. Suzuki, I. Oshima, T. Hirasawa, T. Sakata, T. Horikawa, A. Arimura, Impact of the Gly573Ser substitution in TRPV3 on the development of allergic and pruritic dermatitis in mice, J. Invest. Dermatol. 129 (2009) 714-722.

[20] X. Cheng, J. Jin, L. Hu, D. Shen, X.P. Dong, M.A. Samie, J. Knoff, B. Eisinger, M.L. Liu, S.M. Huang, M.J. Caterina, P. Dempsey, L.E. Michael, A.A. Dlugosz, N.C. Andrews, D.E. Clapham, H. Xu, TRP channel regulates EGFR signaling in hair morphogenesis and skin barrier formation, Cell 141 (2010) 331-343.

[21] E. Yamamoto-Kasai, K. Imura, K. Yasui, M. Shichijou, I. Oshima, T. Hirasawa, T. Sakata, T. Yoshioka, TRPV3 as a therapeutic target for itch, J. Invest. Dermatol. 132 (2012) 2109-2112. 
[22] A. Kihara, Synthesis and degradation pathways, functions, and pathology of ceramides and epidermal acylceramides, Prog. Lipid Res. 63 (2016) 50-69.

[23] K.R. Feingold, P.M. Elias, Role of lipids in the formation and maintenance of the cutaneous permeability barrier, Biochim. Biophys. Acta 1841 (2014) 280-294.

[24] B. Breiden, K. Sandhoff, The role of sphingolipid metabolism in cutaneous permeability barrier formation, Biochim. Biophys. Acta 1841 (2014) 441-452.

[25] Y. Ohno, S. Nakamichi, A. Ohkuni, N. Kamiyama, A. Naoe, H. Tsujimura, U. Yokose, K. Sugiura, J. Ishikawa, M. Akiyama, A. Kihara, Essential role of the cytochrome P450 CYP4F22 in the production of acylceramide, the key lipid for skin permeability barrier formation, Proc. Natl. Acad. Sci. U.S.A. 112 (2015) 7707-7712.

[26] L.G. Luna, Manual of histologic staining methods of the armed forces institute of pathology, 3rd ed, Manual of histologic staining methods of the armed forces institute of pathology, 3rd ed, Blakiston Division, McGraw-Hill, New York, 1968.

[27] T. Sassa, Y. Ohno, S. Suzuki, T. Nomura, C. Nishioka, T. Kashiwagi, T. Hirayama, M. Akiyama, R. Taguchi, H. Shimizu, S. Itohara, A. Kihara, Impaired epidermal permeability barrier in mice lacking Elovl1, the gene responsible for very-long-chain fatty acid production, Mol. Cell. Biol. 33 (2013) 2787-2796.

[28] D. Warde-Farley, S.L. Donaldson, O. Comes, K. Zuberi, R. Badrawi, P. Chao, M. Franz, C. Grouios, F. Kazi, C.T. Lopes, A. Maitland, S. Mostafavi, J. Montojo, Q. Shao, G. Wright, G.D. Bader, Q. Morris, The GeneMANIA prediction server: biological network integration for gene prioritization and predicting gene function, Nucleic Acids Res. 38 (2010) W214-220.

[29] Y. Masukawa, H. Tsujimura, H. Narita, Liquid chromatography-mass spectrometry 
for comprehensive profiling of ceramide molecules in human hair, J. Lipid Res. 47 (2006) 1559-1571.

[30] S. Duchatelet, L. Guibbal, S. de Veer, S. Fraitag, P. Nitschke, M. Zarhrate, C. Bodemer, A. Hovnanian, Olmsted syndrome with erythromelalgia caused by recessive transient receptor potential vanilloid 3 mutations, Br. J. Dermatol. 171 (2014) 675-678.

[31] R.L. Eckert, A.M. Broome, M. Ruse, N. Robinson, D. Ryan, K. Lee, S100 proteins in the epidermis, J. Invest. Dermatol. 123 (2004) 23-33.

[32] Y. Carrier, H.L. Ma, H.E. Ramon, L. Napierata, C. Small, M. O'Toole, D.A. Young, L.A. Fouser, C. Nickerson-Nutter, M. Collins, K. Dunussi-Joannopoulos, Q.G. Medley, Inter-regulation of Th17 cytokines and the IL-36 cytokines in vitro and in vivo: implications in psoriasis pathogenesis, J. Invest. Dermatol. 131 (2011) $2428-2437$.

[33] Y. Ohno, S. Suto, M. Yamanaka, Y. Mizutani, S. Mitsutake, Y. Igarashi, T. Sassa, A. Kihara, ELOVL1 production of C24 acyl-CoAs is linked to C24 sphingolipid synthesis, Proc. Natl. Acad. Sci. U. S. A. 107 (2010) 18439-18444.

[34] A. Kihara, Very long-chain fatty acids: elongation, physiology and related disorders, J. Biochem. 152 (2012) 387-395.

[35] M. Casteels, M. Sniekers, P. Fraccascia, G.P. Mannaerts, P.P. Van Veldhoven, The role of 2-hydroxyacyl-CoA lyase, a thiamin pyrophosphate-dependent enzyme, in the peroxisomal metabolism of 3-methyl-branched fatty acids and 2-hydroxy straight-chain fatty acids, Biochem. Soc. Trans. 35 (2007) 876-880.

[36] A.R. Turkish, A.L. Henneberry, D. Cromley, M. Padamsee, P. Oelkers, H. Bazzi, A.M. Christiano, J.T. Billheimer, S.L. Sturley, Identification of two novel human 
acyl-CoA wax alcohol acyltransferases: members of the diacylglycerol acyltransferase 2 (DGAT2) gene superfamily, J. Biol. Chem. 280 (2005) $14755-14764$.

[37] V.G. Paton, J.E. Shackelford, S.K. Krisans, Cloning and subcellular localization of hamster and rat isopentenyl diphosphate dimethylallyl diphosphate isomerase. A PTS1 motif targets the enzyme to peroxisomes, J. Biol. Chem. 272 (1997) 18945-18950.

[38] A.M. Gregus, D.S. Dumlao, S.C. Wei, P.C. Norris, L.C. Catella, F.G. Meyerstein, M.W. Buczynski, J.J. Steinauer, B.L. Fitzsimmons, T.L. Yaksh, E.A. Dennis, Systematic analysis of rat 12/15-lipoxygenase enzymes reveals critical role for spinal eLOX3 hepoxilin synthase activity in inflammatory hyperalgesia, FASEB J. 27 (2013) 1939-1949.

[39] P. Ternes, S. Franke, U. Zahringer, P. Sperling, E. Heinz, Identification and characterization of a sphingolipid $\Delta 4$-desaturase family, J. Biol. Chem. 277 (2002) 25512-25518.

[40] P. Ebel, S. Imgrund, K. Vom Dorp, K. Hofmann, H. Maier, H. Drake, J. Degen, P. Dormann, M. Eckhardt, T. Franz, K. Willecke, Ceramide synthase 4 deficiency in mice causes lipid alterations in sebum and results in alopecia, Biochem. J. 461 (2014) 147-158.

[41] I. Borbiro, E. Lisztes, B.I. Toth, G. Czifra, A. Olah, A.G. Szollosi, N. Szentandrassy, P.P. Nanasi, Z. Peter, R. Paus, L. Kovacs, T. Biro, Activation of transient receptor potential vanilloid-3 inhibits human hair growth, J. Invest. Dermatol. 131 (2011) 1605-1614.

[42] A.R. Brash, W.E. Boeglin, M.S. Chang, Discovery of a second 15S-lipoxygenase in 
humans, Proc. Natl. Acad. Sci. U.S.A. 94 (1997) 6148-6152.

[43] S.B. Shappell, D.S. Keeney, J. Zhang, R. Page, S.J. Olson, A.R. Brash, 15-Lipoxygenase-2 expression in benign and neoplastic sebaceous glands and other cutaneous adnexa, J. Invest. Dermatol. 117 (2001) 36-43.

[44] D.G. Tang, B. Bhatia, S. Tang, R. Schneider-Broussard, 15-lipoxygenase 2 (15-LOX2) is a functional tumor suppressor that regulates human prostate epithelial cell differentiation, senescence, and growth (size), Prostaglandins Other Lipid Mediat. 82 (2007) 135-146.

[45] A.D. Dobrian, D.C. Lieb, B.K. Cole, D.A. Taylor-Fishwick, S.K. Chakrabarti, J.L. Nadler, Functional and pathological roles of the 12- and 15-lipoxygenases, Prog. Lipid Res. 50 (2011) 115-131.

[46] C.N. Serhan, N. Chiang, T.E. Van Dyke, Resolving inflammation: dual anti-inflammatory and pro-resolution lipid mediators, Nat. Rev. Immunol. 8 (2008) 349-361.

[47] C.N. Serhan, N. Chiang, Endogenous pro-resolving and anti-inflammatory lipid mediators: a new pharmacologic genus, Br. J. Pharmacol. 153 Suppl 1 (2008) S200-215.

[48] M. Romano, E. Cianci, F. Simiele, A. Recchiuti, Lipoxins and aspirin-triggered lipoxins in resolution of inflammation, Eur. J. Pharmacol. 760 (2015) 49-63.

[49] O. Soyombo, B.W. Spur, T.H. Lee, Effects of lipoxin $\mathrm{A}_{4}$ on chemotaxis and degranulation of human eosinophils stimulated by platelet-activating factor and N-formyl-L-methionyl-L-leucyl-L-phenylalanine, Allergy 49 (1994) 230-234.

[50] J.F. Maddox, C.N. Serhan, Lipoxin $A_{4}$ and $B_{4}$ are potent stimuli for human monocyte migration and adhesion: selective inactivation by dehydrogenation and 
reduction, J. Exp. Med. 183 (1996) 137-146.

[51] C. Bandeira-Melo, M.F. Serra, B.L. Diaz, R.S. Cordeiro, P.M. Silva, H.L. Lenzi, Y.S. Bakhle, C.N. Serhan, M.A. Martins, Cyclooxygenase-2-derived prostaglandin $\mathrm{E}_{2}$ and lipoxin $\mathrm{A}_{4}$ accelerate resolution of allergic edema in Angiostrongylus costaricensis-infected rats: relationship with concurrent eosinophilia, J. Immunol. 164 (2000) 1029-1036.

[52] C. Godson, S. Mitchell, K. Harvey, N.A. Petasis, N. Hogg, H.R. Brady, Cutting edge: lipoxins rapidly stimulate nonphlogistic phagocytosis of apoptotic neutrophils by monocyte-derived macrophages, J. Immunol. 164 (2000) 1663-1667.

[53] T.A. Wynn, Cellular and molecular mechanisms of fibrosis, J. Pathol. 214 (2008) 199-210.

[54] Y. Le, Y. Zhou, P. Iribarren, J. Wang, Chemokines and chemokine receptors: their manifold roles in homeostasis and disease, Cell. Mol. Immunol. 1 (2004) 95-104.

[55] M.S. Gresnigt, F.L. van de Veerdonk, Biology of IL-36 cytokines and their role in disease, Semin. Immunol. 25 (2013) 458-465.

[56] H. Blumberg, H. Dinh, E.S. Trueblood, J. Pretorius, D. Kugler, N. Weng, S.T. Kanaly, J.E. Towne, C.R. Willis, M.K. Kuechle, J.E. Sims, J.J. Peschon, Opposing activities of two novel members of the IL-1 ligand family regulate skin inflammation, J. Exp. Med. 204 (2007) 2603-2614.

[57] I.S. Thorey, J. Roth, J. Regenbogen, J.P. Halle, M. Bittner, T. Vogl, S. Kaesler, P. Bugnon, B. Reitmaier, S. Durka, A. Graf, M. Wockner, N. Rieger, A. Konstantinow, E. Wolf, A. Goppelt, S. Werner, The $\mathrm{Ca}^{2+}$-binding proteins S100A8 and S100A9 are encoded by novel injury-regulated genes, J. Biol. Chem. 276 (2001) 35818-35825.

[58] M.A. Grimbaldeston, C.L. Geczy, N. Tedla, J.J. Finlay-Jones, P.H. Hart, S100A8 
induction in keratinocytes by ultraviolet $\mathrm{A}$ irradiation is dependent on reactive oxygen intermediates, J. Invest. Dermatol. 121 (2003) 1168-1174.

[59] A.M. Broome, D. Ryan, R.L. Eckert, S100 protein subcellular localization during epidermal differentiation and psoriasis, J. Histochem. Cytochem. 51 (2003) 675-685. 


\section{Figure legends}

Fig. 1. Infiltration of leukocytes and increased fibrosis in non-lesional skin area of $\mathrm{Ht}$ rats. (A) Representative images of the unaffected dorsal skin areas of WT and $H t$ rats at 25 weeks old. The tissues were subjected to HE, toluidine blue (TB), Luna, and Masson's trichrome (MTC) stainings. TB and Luna stain intracellular granules of mast cells red-violet (yellow arrowheads) and those of eosinophils bright red (green arrowheads), respectively. MTC stains collagen blue. Scale bars, $200 \mu \mathrm{m}$ (for HE, TB, and MTC) and $50 \mu \mathrm{m}$ (for Luna). (B and C) Hydroxyproline (B) and myeloperoxidase (C) assays of the unaffected dorsal skin areas of WT and Ht rats. Values represent the mean per g dry weight \pm SEM from three independent experiments. Statistically significant differences are indicated $\left({ }^{*} P<0.05\right.$, Wilcoxon rank sum test). (D) Electron microscopy of skin sections from unaffected areas of WT and Ht rats at 3 weeks old. Scale bars, $5 \mu \mathrm{m}$. SC, stratum corneum; SG, stratum granulosum.

Fig. 2. Decreases in 15-LOX metabolites and dihydroceramides in non-lesional skin area of Ht rats. (A-D) Lipids were extracted from skin prepared form the unaffected dorsal skin areas of WT and Ht rats and subjected to LC/MS analyses. (A) Plot of the amount of each lipid in the skin of $H t$ rats relative to the amount in WT rats together with the $P$-value (t-test). (B-G) Absolute amounts of the different lipid species in the tissues of WT and Ht rats: fatty acids (B), COX metabolites (C), 5-LOX metabolites (D), 12-LOX metabolites (E), 15-LOX metabolites (F), and ceramides (G). The plotted values represent the mean \pm SEM from three independent experiments. Statistically significant differences are indicated $\left({ }^{*} P<0.05\right.$, Wilcoxon rank sum test). The notation d18:0/20:0 refers to a dihydroceramide with a combination of C18:0 (chain length, C18; 
no double bond) dihydrosphingosine, which has two hydroxyl groups (d), and C20:0 fatty acid. The notations $\mathrm{d} 18: 1$ and t18:0 refer to sphingosine with $\mathrm{C} 18$ and phytosphingosine with C18 chain lengths, respectively. AA, arachidonic acid; ALA, a-linolenic acid; Cer, ceramide; C1P, ceramide 1-phosphate; Chol, cholesterol; DG, diacylglycerol; DGLA, dihomo- $\gamma$-linolenic acid; DHA, docosahexaenoic acid; DHCer, dihydroceramide; EPA, eicosapentaenoic acid; FA, fatty acid; GLA, $\gamma$-linolenic acid; HEPE, hydroxyeicosapentaenoic acid; HexCer, monohexosylceramide; $\mathrm{Hex}_{2} \mathrm{Cer}$, dihexosylceramide; LCB, long-chain base; LPA, lysophosphatidic acid; LPC, lysophosphatidylcholine; $\quad$ LPE, lysophosphatidylethanolamine; $\quad$ LPG, lysophosphatidylglycerol; LPI, lysophosphatidylinositol; LPS, lysophosphatidylserine; MG, monoacylglycerol; NAE, $N$-acylethanolamine; NAGly, $N$-acylglycine; oxFA, oxidized fatty acid; PA, phosphatidic acid; PC, phosphatidylcholine; PE, phosphatidylethanolamine; PG, prostaglandin; Pgly, phosphatidylglycerol; PI, phosphatidylinositol; PS, phosphatidylserine; SM, sphingomyelin; TG, triacylglycerol.

Fig. 3. 15-LOX metabolites exist in upper skin region. The dorsal skin of unaffected areas of WT and Ht rats were separated into upper (hair and epidermis) and lower (dermis, subcutaneous tissue, and muscle) parts, and lipids were extracted. (A-D) Relative amounts of the 15-LOX (A), 12-LOX (B), 15-LOX (C), and COX (D) metabolites, measured by LC/MS. The plotted values represent the mean \pm SEM from three independent experiments.

Fig. 4. Normal levels of 15-LOX metabolites and dihydroceramides in brain tissue of $\mathrm{Ht}$ rats. Lipids were extracted from the brains of WT and $H t$ rats, and levels of 15-LOX 
metabolites (A) and dihydroceramides (B) were measured by LC/MS. The plotted values represent the mean \pm SEM from three independent experiments.

Fig. 5. Decreases in expression levels of 15-LOX genes in unaffected skin area of $\mathrm{Ht}$ rats. Total RNAs were prepared from the unaffected dorsal skin area of WT and Ht rats and subjected to real-time RT-PCR using primers specific to the indicated genes and hypoxanthine phosphoribosyltransferase 1 (Hprt1) for control. The plotted values represent the mean \pm SEM of the gene expression levels relative to Hprt1 from three independent experiments. Statistically significant differences are indicated $\left({ }^{*} P<0.05\right.$, Wilcoxon rank sum test).

Fig. 6. Model of OS pathogenesis. Constitutive activation of TRPV3 down-regulates expression of Alox15 and Alox15b, which leads to decreased levels of 15-LOX metabolites. TRPV3 activation also increases expression of chemokines and cytokines including Cxcl1, Cxcl2, Ccl2, and Il36b, which in turn leads to leukocyte infiltration. The decrease in 15-LOX metabolites, as well as leukocyte infiltration, induces inflammation, triggering OS pathogenesis. 
Figure 1

\section{A}

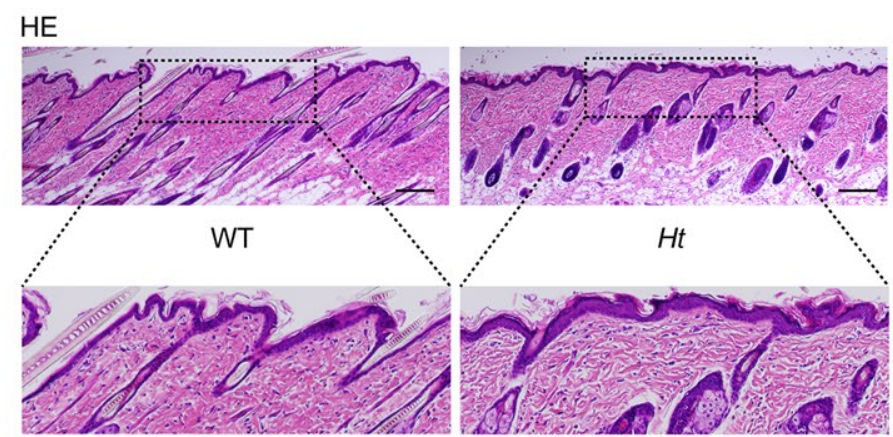

Luna

TB

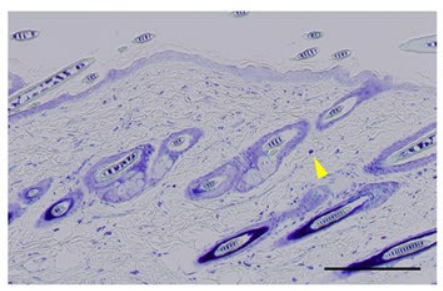

WT

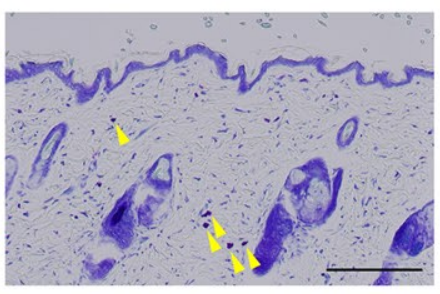

Ht

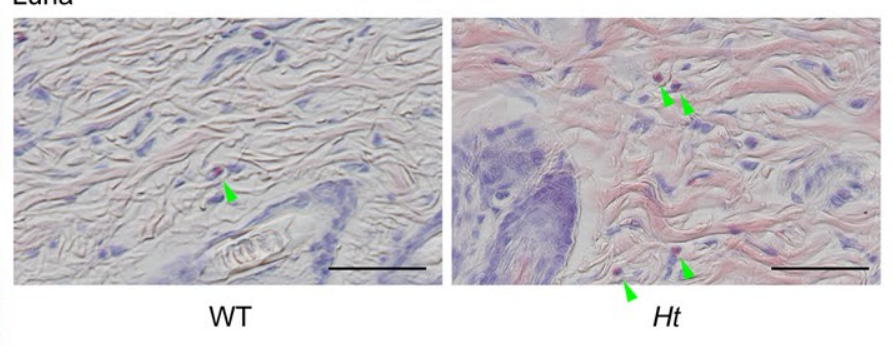
MTC

B

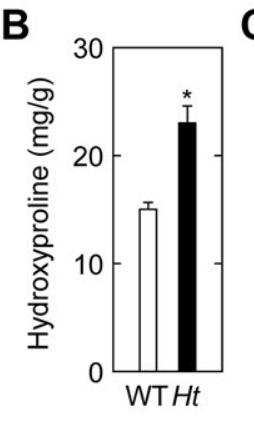

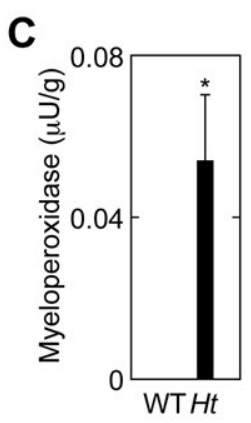

D
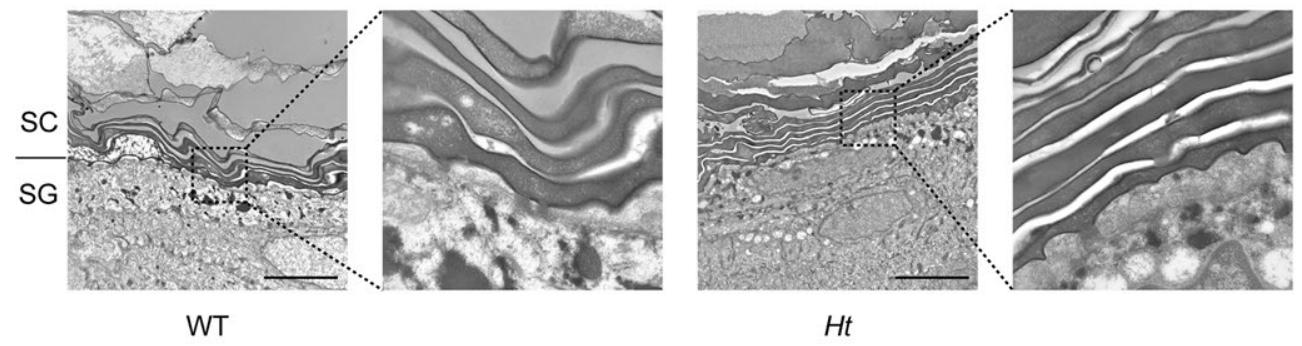
Figure 2
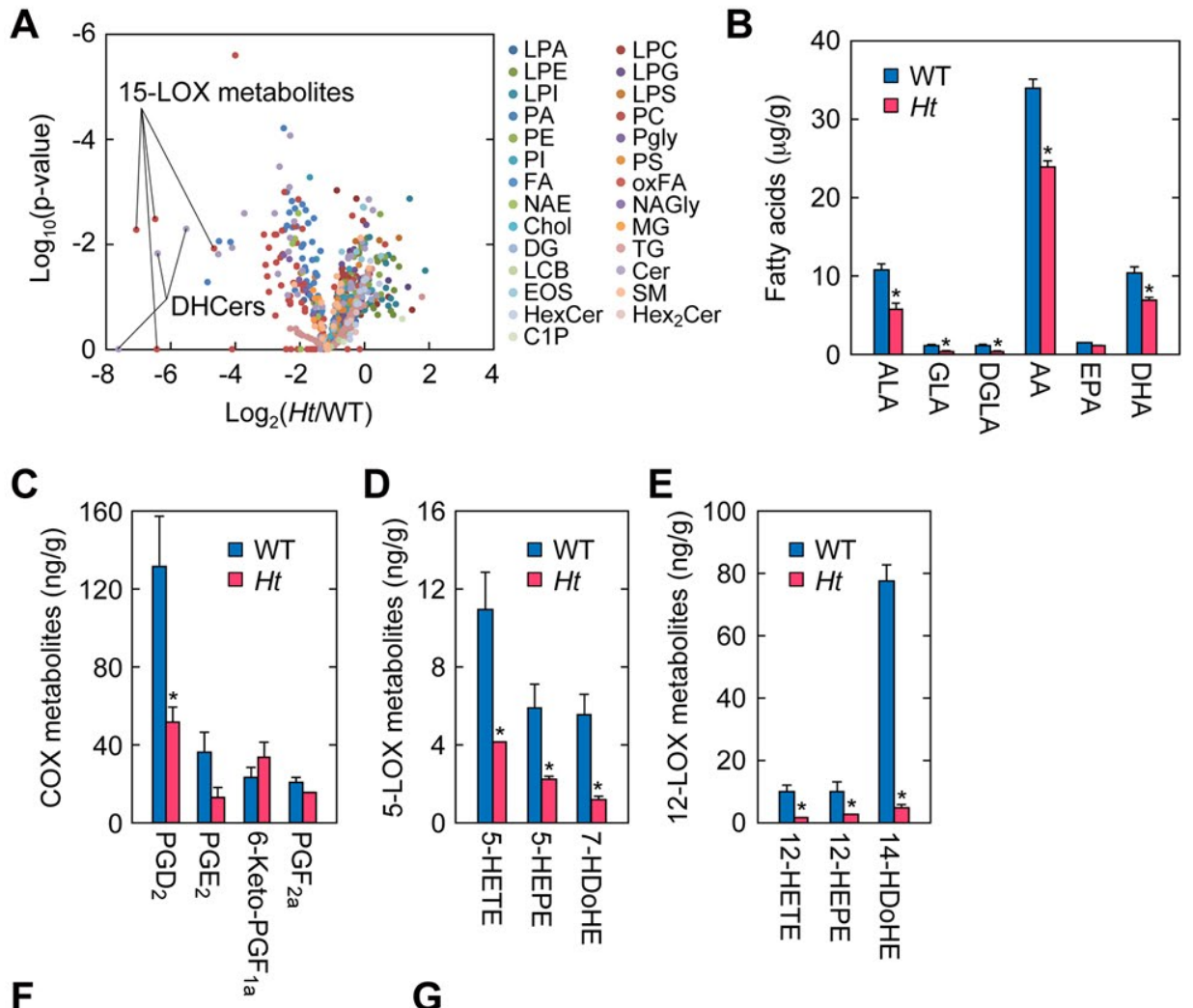

D
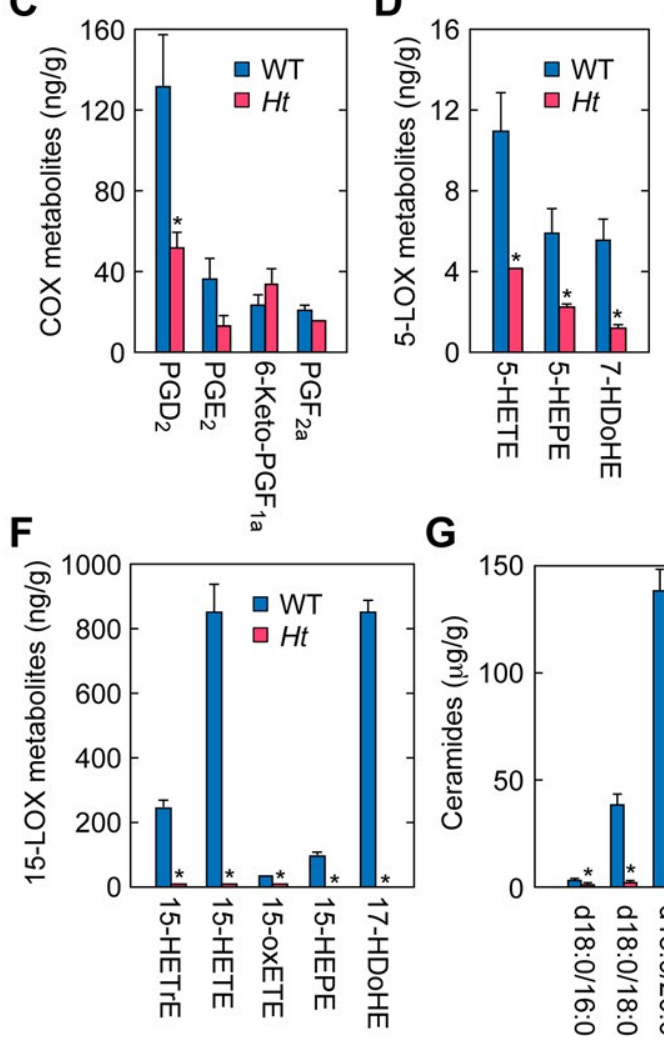

E

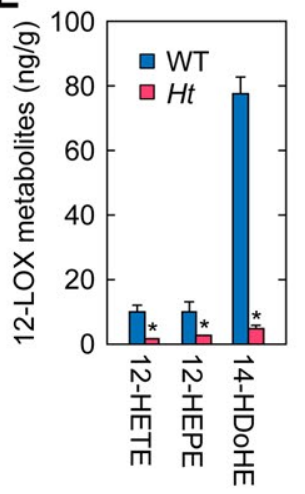

G

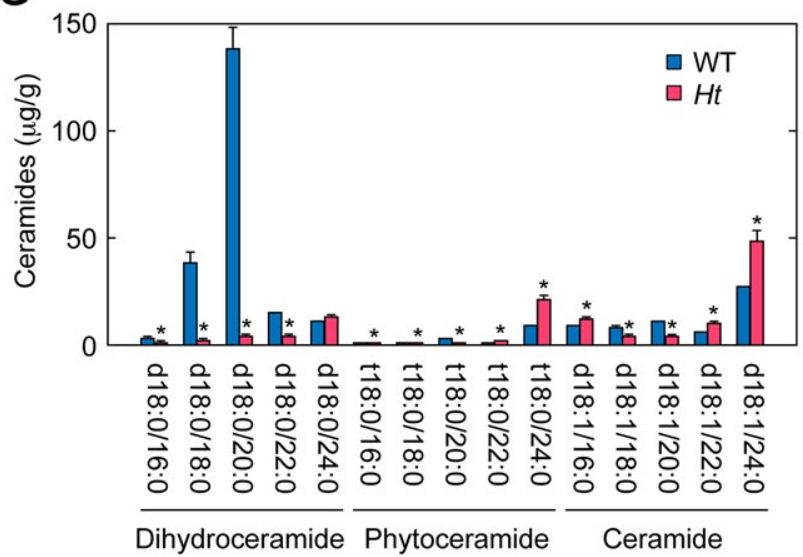


Figure 3

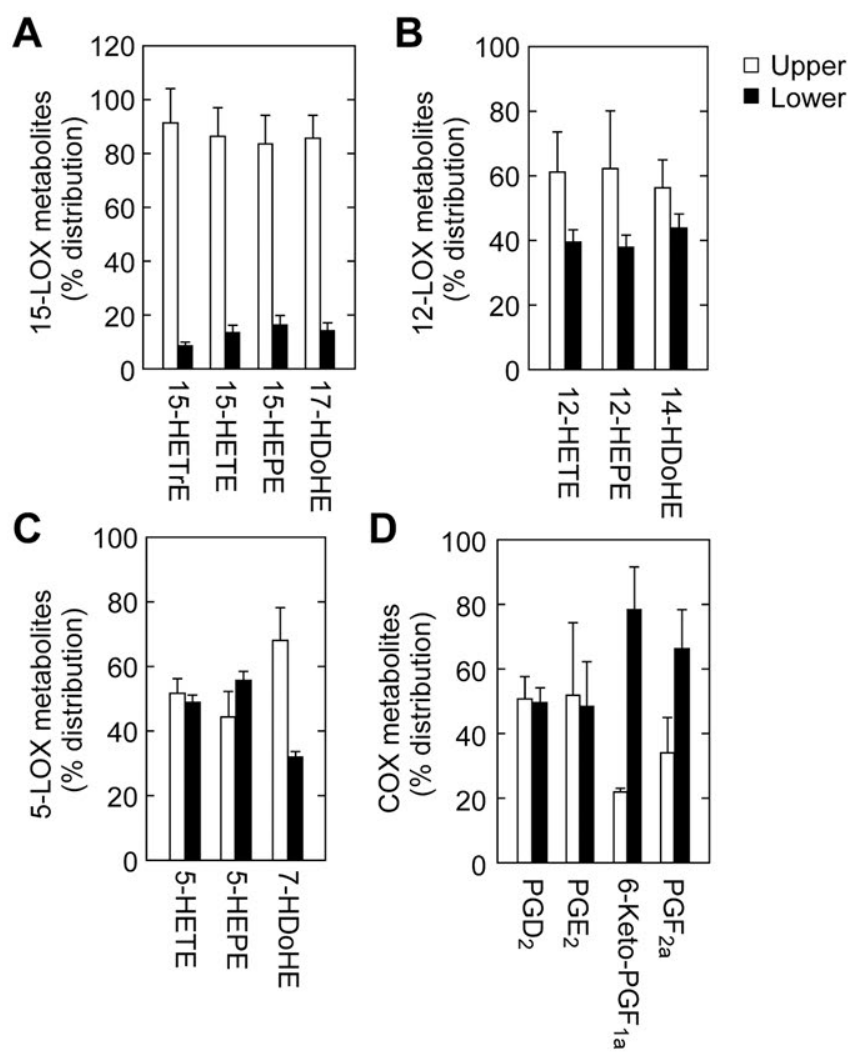


Figure 4

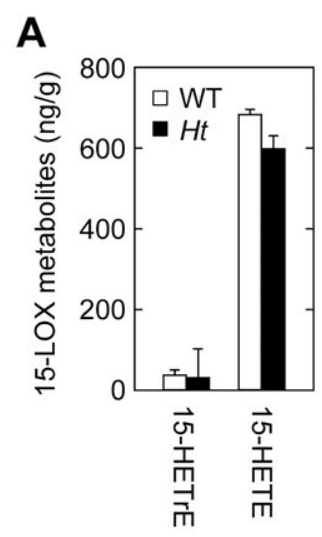

B

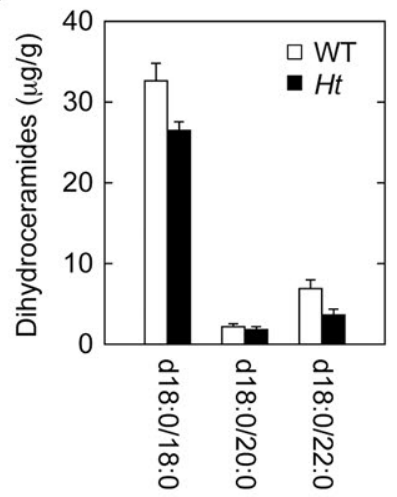


Figure 5
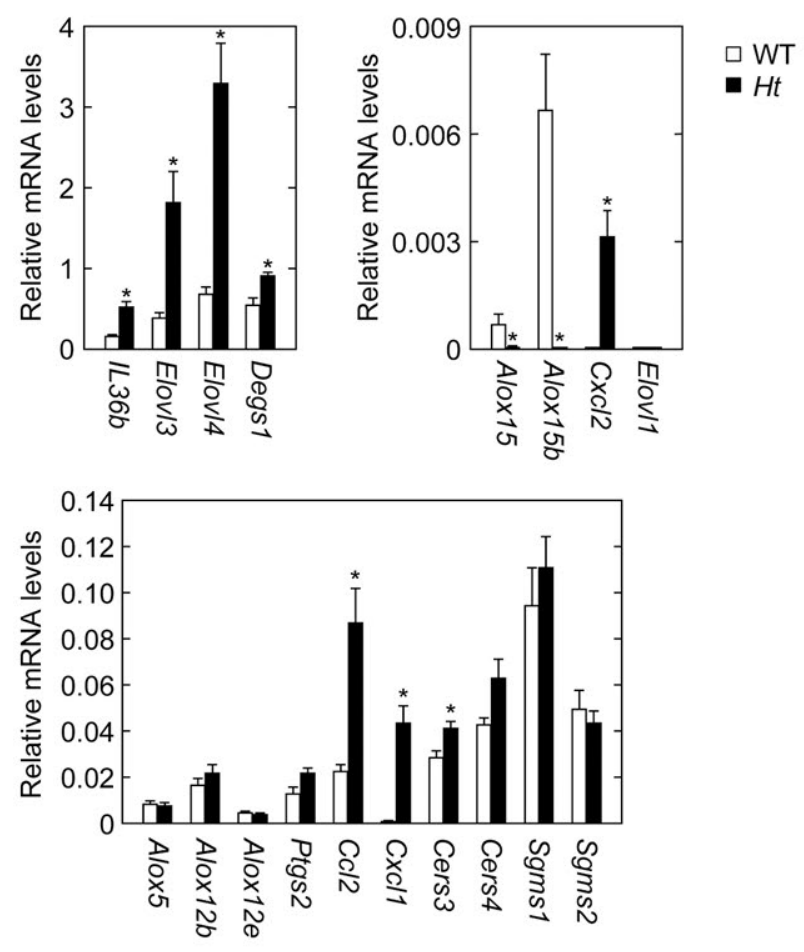
Figure 6

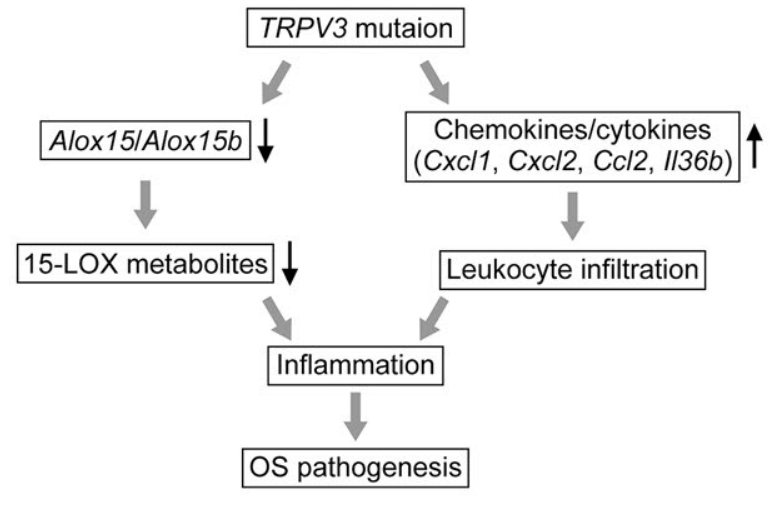




\section{Supplementary Method}

\section{Lipid analyses by $L C / M S$}

Lipids were extracted from skin or brain samples as described previously, with minor modifications [1-3]. Briefly, skin samples were prepared using 8-mm disposable biopsy punches (Kai industries, Gifu, Japan). To measure fatty acid metabolites from two parts of the skin (the upper part containing hair and epidermis, and the lower part containing dermis, subcutaneous tissues, and muscle), skin tissue was incubated with dispase solution $(2,000 \mathrm{U} / \mathrm{ml}$ DISPASE I (Wako Pure Chemical Industries) in Dulbecco's Modified Eagle's Medium containing $10 \%$ fetal bovine serum) at $4{ }^{\circ} \mathrm{C}$ for $16 \mathrm{~h}$, followed by dissection. Samples $(5-50 \mathrm{mg})$ were freeze-dried, chopped, and suspended in $1 \mathrm{~mL}$ of methanol for extraction of fatty acids, fatty acid metabolites, and monoacylglycerols, or in $1 \mathrm{~mL}$ of methanol/methyl-tert-butyl ether/chloroform $(4: 3: 3$, $\mathrm{v} / \mathrm{v}$ ) for extraction of glycerolipids other than monoacylglycerols, sphingolipids, and cholesterol. Both suspension liquids contained $10 \mathrm{mM}$ dibutylhydroxytoluene and internal standards (Tables S1 and S2). Lipids were extracted by vigorously mixing the samples with zirconia beads using a Micro Smash MS-100R (Tomy Seiko, Tokyo, Japan) and recovered by centrifugation. Lipid extraction was repeated, and the two resultant supernatants were pooled. Lipids were dried under a gentle stream of nitrogen gas and suspended in $200 \mu \mathrm{L}$ of methanol (for fatty acids, fatty acid metabolites, and monoacylglycerols) or $250 \mu \mathrm{L}$ of chloroform/methanol (1:1, v/v) (for glycerolipids other than monoacylglycerols, sphingolipids, and cholesterol). The lipid-containing methanol suspension was loaded onto a MonoSpin C18-AX column (GL Sciences, Tokyo, Japan), and the lipids were eluted with methanol/formic acid (50:1, v/v), dried by nitrogen gas, and resuspended in $250 \mu \mathrm{L}$ of methanol. 
Fatty acids, glycerolipids other than monoacylglycerols, sphingolipids, and cholesterol were measured by non-targeted LC/MS analyses essentially as described previously [3,4], using an Ultimate 3000 RSLC system (Thermo Fisher Scientific, Rockford, IL) and a Q-Exactive plus hybrid quadrupole-Orbitrap mass spectrometer (Thermo Fisher Scientific). Lipids were separated using an ACQUITY UPLC BEH C18 column $(100 \mathrm{~mm} \times 2.1 \mathrm{~mm}$; particle size, $1.7 \mu \mathrm{m}$; Waters, Milford, MA) with a flow rate of $0.4 \mathrm{~mL} / \mathrm{min}$ in the binary gradient systems. The mobile phases and gradient steps used were as follows: for fatty acids, mobile phase A (water $/ 5 \mathrm{mM}$ phosphoric acid/acetic acid, 1000:1:1, v/v) and mobile phase B (acetonitrile/isopropanol/acetic acid, $500: 500: 1, \mathrm{v} / \mathrm{v}$ ) from 0 to $43 \mathrm{~min}$ with an elution gradient from $25 \%$ to $100 \% \mathrm{~B}$, from 43 to 48 min with $100 \%$ B, and from 48 to 50 min with $25 \%$ B. For glycerolipids other than monoacylglycerols, sphingolipids, and cholesterol, the LC conditions were as follows: mobile phase $\mathrm{A}$ (water/1 $\mathrm{M}$ ammonium acetate $/ 5 \mathrm{mM}$ phosphoric acid/formic acid, 990:10:1:1, v/v) and mobile phase B (acetonitrile/isopropanol/1 M ammonium acetate/formic acid, 495:495:10:1, v/v) from 0 to 43 min with an elution gradient from $50 \%$ to $100 \% \mathrm{~B}$, from 43 to 48 min with $100 \% \mathrm{~B}$, and from 48 to 50 min with $50 \% \mathrm{~B}$. Fatty acids, glycerophospholipids/lyso-glycerophospholipids of glycerolipids, and sphingomyelins of sphingolipids were detected in the negative electrospray ionization mode, whereas triacylglycerols/diacylglycerols of glycerolipids and ceramide 1-phosphates/monohexosylceramides/dihexosylceramides/ceramides/acylceramides (EOS, esterified $\omega$-hydroxyacyl sphingosine)/long-chain bases of sphingolipids were detected in the positive electrospray ionization mode. Cholesterol was detected in the positive atmospheric pressure chemical ionization mode. Lipids were identified using the Xcalibur 3.0 Qual Browser (Thermo Fisher Scientific) and Lipid Search software 
(Mitsui Knowledge Industry, Tokyo, Japan) [5]. The mass tolerance was set to a 10-ppm mass window. Target lipids were identified as $[\mathrm{M}-\mathrm{H}]^{-}$ion (for fatty acids, phosphatidic acids, phosphatidylethanolamines, phosphatidylglycerols, phosphatidylinositols, phosphatidylserines, lysophosphatidic acids, lysophosphatidylethanolamines, lysophosphatidylglycerols, lysophosphatidylinositols, and lysophosphatidylserines), $[\mathrm{M}+\mathrm{HCOO}]^{-}$ion (for phosphatidylcholines, lysophosphatidylcholines, and sphingomyelins), $\left[\mathrm{M}+\mathrm{NH}_{4}\right]^{+}$ion (for triacylglycerols and diacylglycerols), $[\mathrm{M}+\mathrm{H}]^{+}$ion (for long-chain bases, ceramide 1-phosphates, monohexosylceramides, dihexosylceramides, acylceramides, and ceramides), or $\left[\mathrm{M}-\mathrm{H}_{2} \mathrm{O}+\mathrm{H}\right]^{+}$ion (for cholesterol). For quantification, calibration curves were created from 5 to 5,000 or $10,000 \mathrm{ng} / \mathrm{mL}$ using the lipids listed in Table $\mathrm{S} 3$, and the regions of the calibration curves that showed linearity were used for quantification. The lipids, except for the acylceramide EOS, were quantified by absolute quantification using the corresponding standard lipids or by relative quantification using the standards of related lipids (Table S3). For the acylceramide EOS, which is not commercially available, quantification was performed by comparison with the peak area of the internal standard (ceramide $\left.(\mathrm{d} 18: 1 / 16: 0)-\mathrm{d}_{31}\right)$.

Fatty acid metabolites and monoacylglycerols were analyzed by targeted LC/MS/MS, as described previously [2,3], using an ACQUITY UPLC system (Waters) and API5000 triple quadrupole mass spectrometer (AB SCIEX, Framingham, MA). Lipids were separated by high-performance liquid chromatography on a reverse-phase column (ACQUITY UPLC BEH C18 column; $100 \times 2.1 \mathrm{~mm}$; particle size, $1.7 \mu \mathrm{m}$; Waters) and detected by MS. The flow rate was $0.4 \mathrm{~mL} / \mathrm{min}$ in a binary gradient system using a mobile phase A (water/5 mM phosphoric acid/acetic acid, 1000:1:1, v/v) and a 
mobile phase B (acetonitrile/isopropanol/acetic acid, 500:500:1, v/v). The elution gradient steps were as follows: from 0 to $15 \mathrm{~min}$ with an elution gradient from $25 \%$ to $60 \% \mathrm{~B}$, from 15 to 17.5 min with $100 \% \mathrm{~B}$, and from 17.5 to 20 min with $25 \% \mathrm{~B}$. Lipids were detected by multiple reaction monitoring in a negative (for oxidized fatty acids and $N$-acylglycines) or positive (for $N$-acylethanolamines and monoacylglycerols) ion mode. Calibration curves were created from 0.03 to $100 \mathrm{ng} / \mathrm{mL}$ using the lipids listed in Table S4, and the regions of the calibration curves that showed linearity were used for quantification. 


\section{Supplementary References}

[1] R.M. Pellegrino, A. Di Veroli, A. Valeri, L. Goracci, G. Cruciani, LC/MS lipid profiling from human serum: a new method for global lipid extraction, Anal. Bioanal. Chem. 406 (2014) 7937-7948.

[2] T. Sanaki, T. Fujiwara, R. Iwamoto, T. Yoshioka, K. Higashino, T. Nakano, Y. Numata, Improvements in the high-performance liquid chromatography and extraction conditions for the analysis of oxidized fatty acids using a mixed-mode spin column, Mod. Chem. Appl. 3 (2015) 1000161.

[3] T. Sanaki, Y. Inaba, T. Fujiwara, T. Yoshioka, K. Matsushima, K. Minagawa, K. Higashino, T. Nakano, Y. Numata, A hybrid strategy using global analysis of oxidized fatty acids and bioconversion by Bacillus circulans, Rapid Commun. Mass Spectrom. 30 (2016) 751-762.

[4] M. Sugimoto, Y. Shimizu, T. Yoshioka, M. Wakabayashi, Y. Tanaka, K. Higashino, Y. Numata, S. Sakai, A. Kihara, Y. Igarashi, Y. Kuge, Histological analyses by matrix-assisted laser desorption/ionization-imaging mass spectrometry reveal differential localization of sphingomyelin molecular species regulated by particular ceramide synthase in mouse brains, Biochim. Biophys. Acta 1851 (2015) $1554-1565$.

[5] T. Yamada, T. Uchikata, S. Sakamoto, Y. Yokoi, E. Fukusaki, T. Bamba, Development of a lipid profiling system using reverse-phase liquid chromatography coupled to high-resolution mass spectrometry with rapid polarity switching and an automated lipid identification software, J. Chromatogr. A 1292 (2013) 211-218. 


\section{Supplementary Figure Legends}

Fig. S1. Structures of ceramide species found in the epidermis. Structures of dihydroceramide, ceramide, phytoceramide, and the acylceramide EOS are illustrated.

Fig. S2. Hyperkeratosis, parakeratosis, and acanthosis in the affected area of $H t$ rat skin. Unaffected skin of WT and affected skin of $H t$ rats at 25 weeks old were subjected to HE staining. Blue and pink arrowheads indicate monocytes and granulated leukocytes, respectively. Black dotted line indicates the boundary between epidermis and dermis. Scale bars, $500 \mu \mathrm{m}$.

Fig. S3. Metabolic pathways of polyunsaturated fatty acids (PUFAs). Metabolic pathways of n-6 and n-3 PUFAs are illustrated. AA, arachidonic acid; ALA, $\alpha$-linolenic acid; COX, cyclooxygenase; DGLA, dihomo- $\gamma$-linolenic acid; DHA, docosahexaenoic acid; EPA, eicosapentaenoic acid; GLA, $\gamma$-linolenic acid; LA, linoleic acid; LT, leukotriene; LX, lipoxin; MaR, maresin; PD, protectin D; PG, prostaglandin; Rv, resolvin.

Fig. S4. Keratin- and cytoskeleton-related genes are down-regulated in the unaffected dorsal skin tissue of $H t$ rats. The top 30 genes that were found to be down-regulated in the unaffected skin tissue of $H t$ rats compared with WT rats by microarray analyses (Table 1) were subjected to gene function prediction using GeneMANIA (http://www.genemania.org/). FDR, false discovery rate.

Fig. S5. Leukocyte chemotaxis-related genes are up-regulated in the unaffected dorsal 
skin tissue of $H t$ rats. The top 30 genes that were found to be up-regulated in the unaffected skin tissue of $H t$ rats compared with WT rats by microarray analyses (Table 2) were subjected to gene function prediction using GeneMANIA (http://www.genemania.org/). FDR, false discovery rate.

Fig. S6. Lipid metabolism-related genes are up-regulated in the unaffected dorsal skin tissue of $H t$ rats. The top 30 genes that were found to be highly expressed in $H t$ rats among genes exhibiting increased expression ( $>1.5$ fold increase, $\left.\log _{2} H t / \mathrm{WT}\right)$ by microarray analyses (Table 3) were subjected to gene function prediction using GeneMANIA (http://www.genemania.org/). FDR, false discovery rate.

Fig. S7. Expression levels of Alox 15 and Alox $15 b$ in brain. Total RNAs were prepared from the brains of WT and $H t$ rats and subjected to real-time RT-PCR using primers specific to Alox 15, Alox15b, and glyceraldehyde-3-phosphate dehydrogenase (Gapdh) for control. The plotted values represent the gene expression levels relative to Gapdh. 
Figure S1
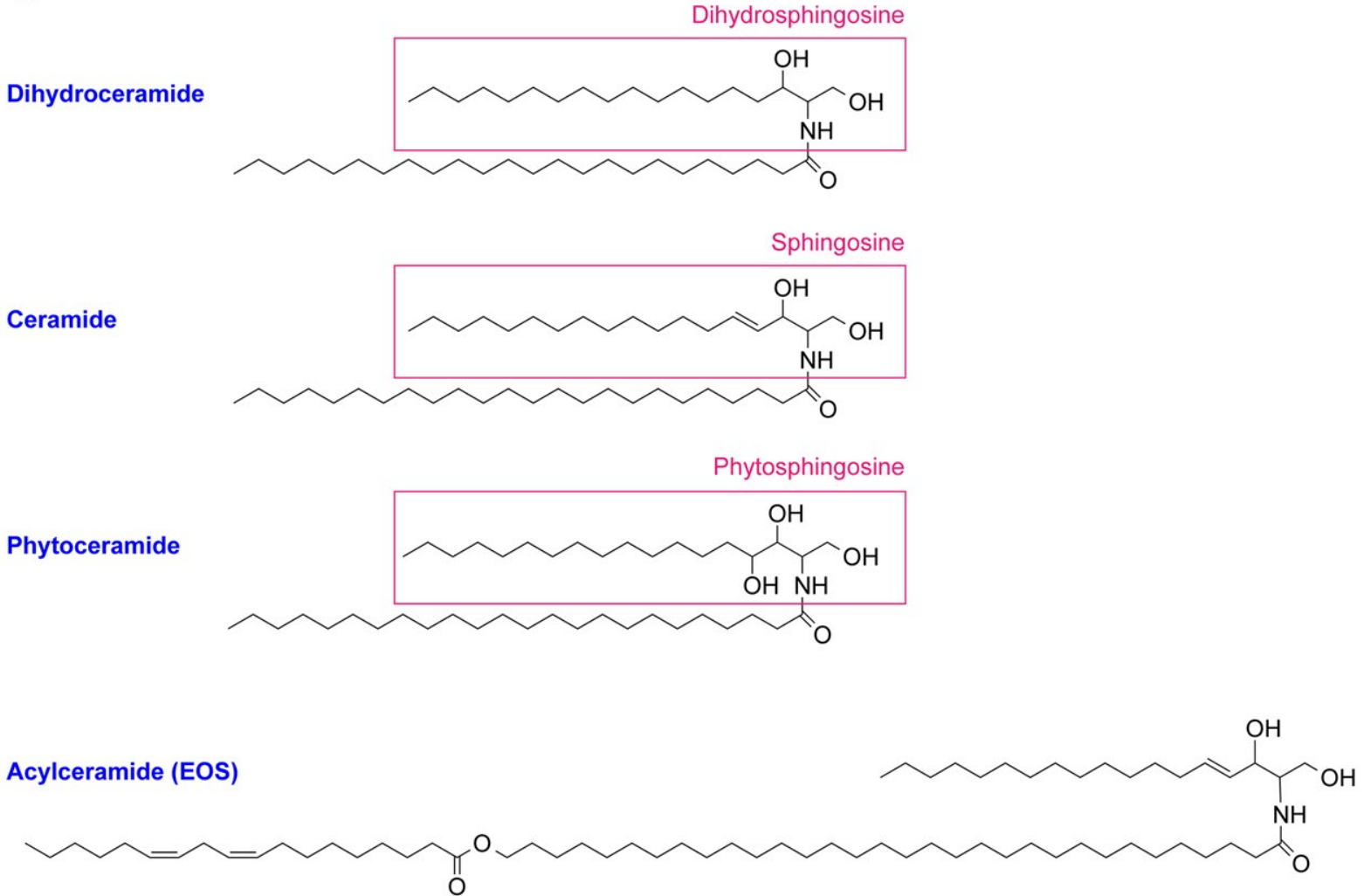
Figure S2
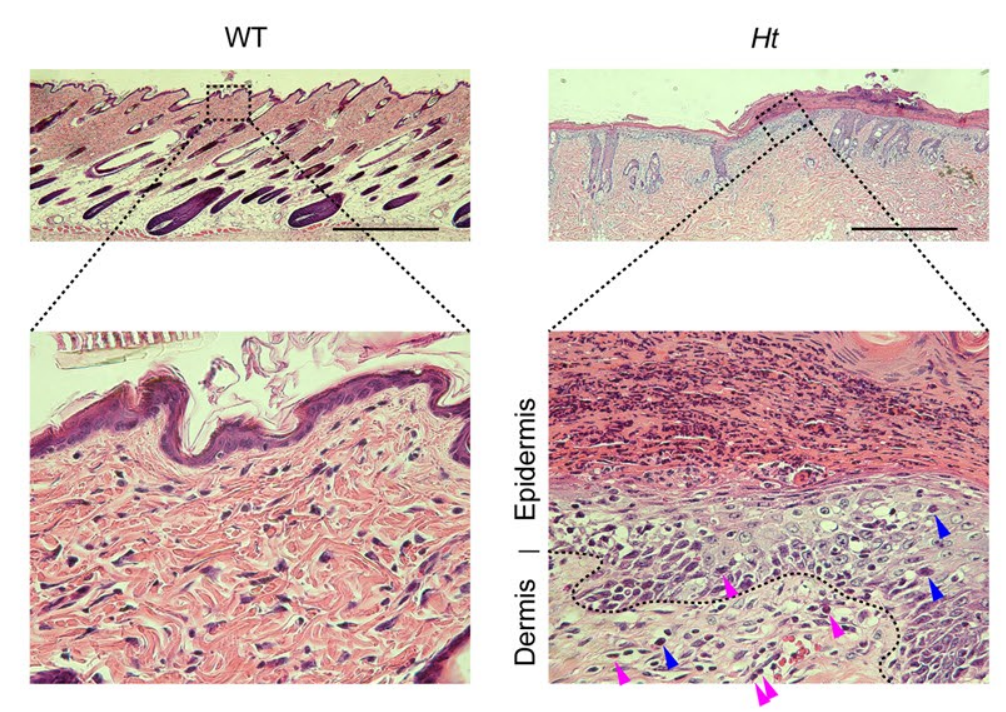
Figure S3

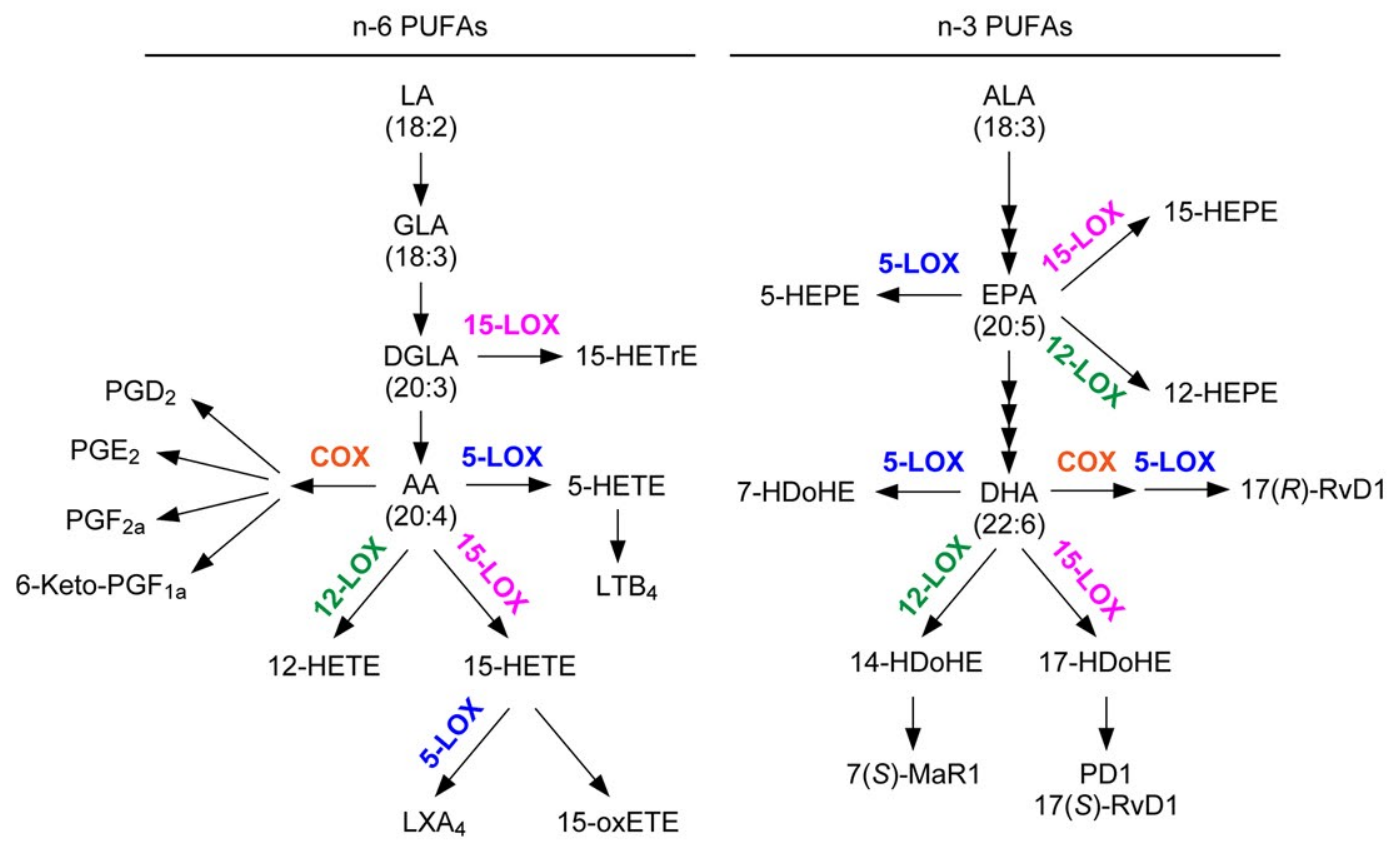


Figure S4

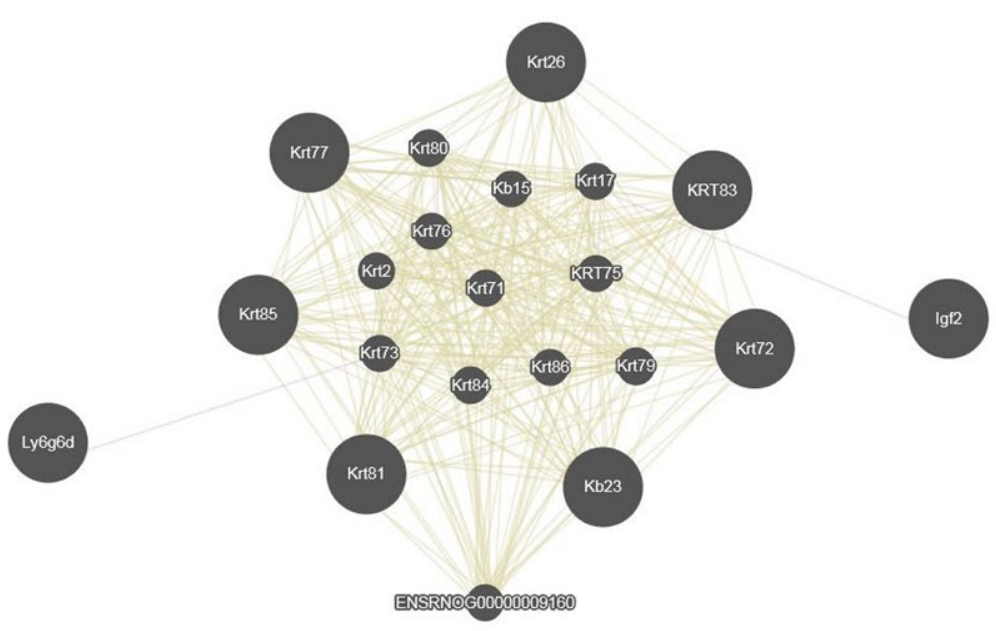

\begin{tabular}{lccc}
\hline Function & FDR & Genes in network & Genes in genome \\
\hline Keratin filament & $1.05 \times 10^{-5}$ & 5 & 21 \\
Intermediate filament cytoskeleton & $4.04 \times 10^{-4}$ & 6 & 97 \\
Intermediate filament & $5.19 \times 10^{-4}$ & 5 & 54 \\
\hline
\end{tabular}




\section{Figure S5}

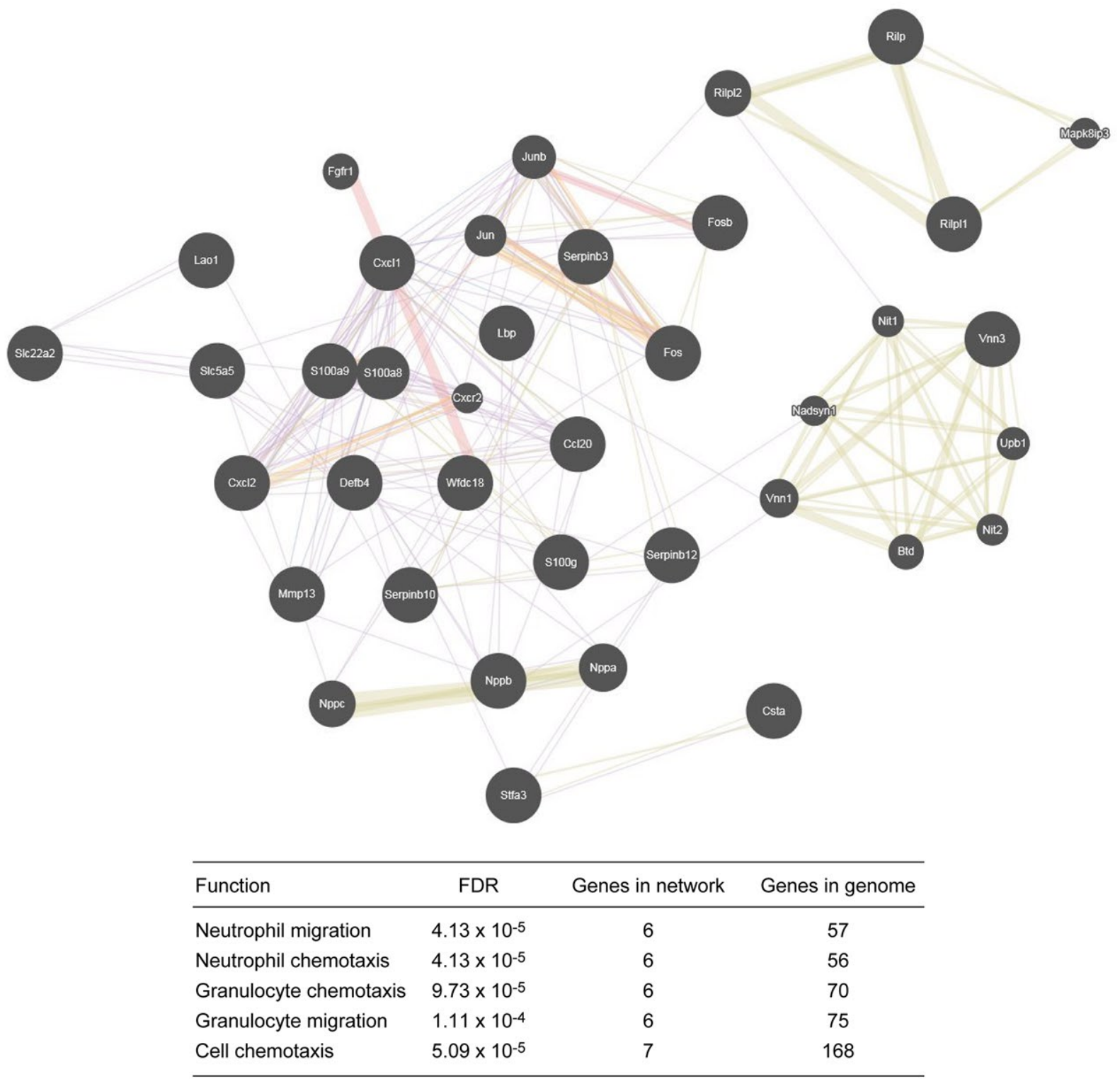




\section{Figure S6}

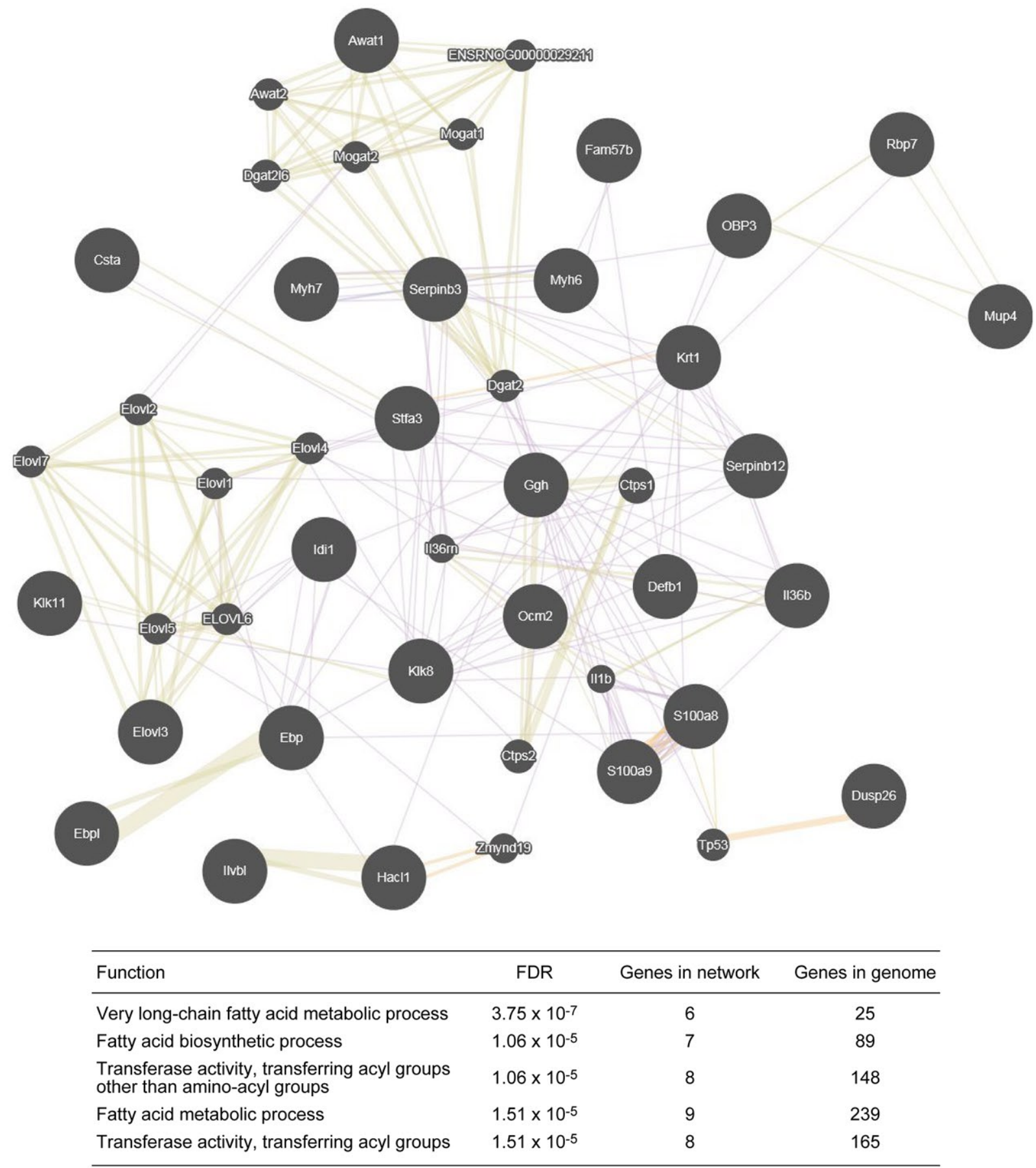


Figure S7

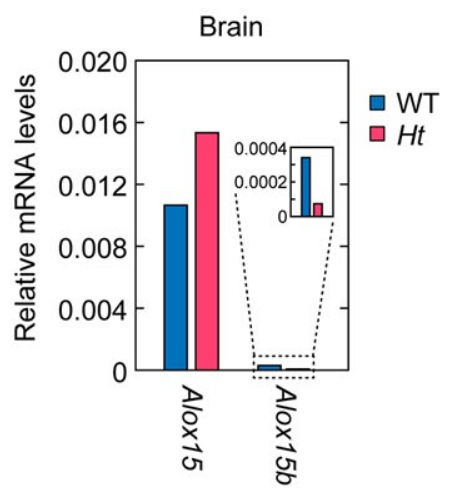

